\title{
Relação entre a vegetação e as propriedades do solo em áreas de campo limpo úmido no Parque Nacional de Sete Cidades, Piauí, Brasil
}

Vegetation and soil relationship in moist grassland in the National Park of Sete Cidades, Piauí, Brazil

\author{
Maura Rejane de Araújo Mendes ${ }^{1,5}$, Cássia Beatriz Rodrigues Munhoz ${ }^{2}$, \\ Manoel Cláudio da Silva Júnior ${ }^{3} \&$ Antonio Alberto Jorge Farias Castro ${ }^{4}$
}

\begin{abstract}
Resumo
O objetivo desse estudo foi avaliar a estrutura da camada herbáceo-subarbustiva em comunidades de campo limpo no Parque Nacional de Sete Cidades localizado no Piauí, e a sua relação com as variáveis edáficas e espaciais das áreas. A amostragem da vegetação foi realizada no final da estação chuvosa/início da seca, através do método de interseção na linha, para determinar a composição e a cobertura linear das espécies. Dezessete linhas de $10 \mathrm{~m}$, subdivididas em seções de $1 \mathrm{~m}$, foram distribuídas em seis áreas distintas. Amostras de solo $(0-20 \mathrm{~cm})$ foram coletadas para análises químicas e texturais. Foram amostrados 71 táxons relacionados a 46 gêneros e 25 famílias. A análise de correspondência canônica (CCA) separou as linhas amostradas em três grupos, associados as propriedades texturais e químicas do solos, que coincidiram com as áreas mais similares. O procedimento de permutação com múltiplas respostas (MRPP) detectou diferenças significativas entre os grupos da CCA. As variáveis ambientais mais fortemente correlacionadas foram $\mathrm{Cu}, \mathrm{Fe}$, saturação de alumínio, silte, Zn, saturação de bases e areia, explicando 37,9\% da variação dos dados. A distância espacial entre as linhas foi responsável por 16,8\% da variação explicada. A distribuição dos campos úmidos no Parque parece estar determinada pelos gradientes de textura e fertilidade dos solos.

Palavras-chave: cerrado, estrato herbáceo, método de interseção na linha, variáveis ambientais.
\end{abstract}

\begin{abstract}
Variations in soil proprieties seem to determine the spatial distribution of plant communities in the moist grassland. The aim of this study was to evaluate the relationship between the floristic composition and structure of the herbaceous-subshrub layer and soil and spatial variables in grassland communities in the National Park of Sete Cidades, Piauí, Brazil. The composition and linear coverage of the species were assessed by the lineintercept method. A total of 17 lines of $10 \mathrm{~m}$, subdivided in sections of $1 \mathrm{~m}$, were distributed into six distinct areas. Soil samples were collected for chemical and textural analysis. We sampled 71 taxa in 46 genera and 25 families. Through canonical correspondence analysis (CCA) the lines sampled were separated into three groups, associated with the textural and chemical soil properties, which coincided with the most similar sites. The multi-response permutation procedures (MRPP) found significant differences between groups of the CCA. The environmental variables most strongly correlated were $\mathrm{Cu}, \mathrm{Fe}$, aluminum saturation, silt, $\mathrm{Zn}$, base saturation and sand, accounting for $37.9 \%$ of data variation. The spatial distance between the lines accounted for $16.8 \%$ of the explained variance. The distribution of the wet grasslands in the park seems to be determined by texture and soil fertility gradients.
\end{abstract}

Key words: cerrado, herbaceous layer, line-intercept method, environmental variables

Este artigo possui material adicional em sua versão eletrônica.

\footnotetext{
${ }^{1}$ Universidade Estadual do Piauí, 64202-220, Parnaíba, PI, Brasil.

${ }^{2}$ Universidade de Brasília, Depto. Botânica, 70910-900, Brasília, DF, Brasil.

${ }^{3}$ Universidade de Brasília, Depto. Engenharia Florestal, 71919-970, Brasília, DF, Brasil.

${ }^{4}$ Universidade Federal do Piauí, Depto. Biologia, 64049-550, Teresina, PI, Brasil.

${ }^{5}$ Autora para correspondência: maurarejanem@gmail.com
} 


\section{Introdução}

O solo é considerado como principal fator ecológico na determinação da ocorrência de savanas, cujas explicações edáficas incluem especialmente os teores de nutrientes e a umidade (Montgomery \& Askew 1983). No Cerrado, a heterogeneidade na paisagem, com a sucessão de diferentes formações fisionômicas, em estudos iniciais foi associada com diferenças na fertilidade, níveis elevados de alumínio e acidez no solo (Alvim \& Araújo 1952; Arens 1958). Entretanto, estudos posteriores mostraram resultados conflitantes para a explicação das relações entre fisionomias e status nutricional dos solos (Furley \& Ratter 1988; Ruggiero et al. 2002). De modo geral, além da baixa fertilidade dos solos comum para todas as fisionomias (Furley \& Ratter 1988), outros fatores edáficos desempenham papel fundamental na distribuição das mesmas, como a profundidade do solo, a presença de concreções, e outros obstáculos que impeçam o desenvolvimento das raízes, além da água (Haridasan 2008).

Ao longo do gradiente físionômico podem ocorrer variações na identidade das espécies e nos processos desses ecossistemas naturais (Haridasan 2000). Estudos indicam que áreas com solos mais férteis apresentam diferenças na composição florística, densidade das espécies dominantes e concentrações de nutrientes nas folhas, e que a menor fertilidade seleciona espécies com baixo requerimento nutricional (Haridasan 2000; Moreno et al. 2007).

Espécies do Cerrado possuem alguns mecanismos adaptativos que as tornam capazes de lidar com deficiências nutricionais no solo. No entanto são capazes de responder positivamente a disponibilidade de recursos, como nutrientes, água, luz e ausência de competição (Haridasan 2008). Por exemplo, disponibilidade de fósforo no solo pode regular a frequência e a produtividade de Leguminosas, determinando sua maior ocorrência em áreas mais secas (Medina 1996; Janssens et al. 1998; Amorim \& Batalha 2007), enquanto o excesso de umidade está associado ao aparecimento de espécies de Cyperaceae, Xyridaceae, Eriocaulaceae e Lentibulariaceae (Tannus \& Assis 2004; Munhoz \& Felfili 2006b, 2007). Mesmo em áreas adjacentes, ocorre uma acentuada substituição de espécies entre os campos úmidos e secos (Tannus \& Assis 2004).
Dentro da mesma área campestre tropical, variações nas propriedades do solo, nos teores de matéria orgânica, alumínio, argila, saturação de alumínio e fósforo foram correlacionados com a estrutura das comunidades de plantas (Amorim \& Batalha 2006; Munhoz et al. 2008). Amorim \& Batalha (2008) associaram a elevação na riqueza de espécies em áreas do Parque Nacional das Emas, Goiás com elevado teor de alumínio e baixo $\mathrm{pH}$. Em veredas, ambientes caracterizados por solos hidromórficos, variações locais na umidade determinam aumento de espécies das áreas mais úmidas para as mais secas (Guimarães et al. 2002; Ramos et al. 2006), e mais espécies exclusivas nas extremidades de umidade da vereda (Araújo et al. 2002).

Pela carência de estudos que abordam as relações entre o solo e a vegetação em áreas de campo limpo no Cerrado do Nordeste, este trabalho objetiva avaliar áreas de campo limpo no Parque Nacional de Sete Cidades (ParnaSC), uma área de transição Cerrado/Caatinga, localizado na porção norte do Estado do Piauí, e submetido à forte heterogeneidade das condições ambientais. Tendo como premissa que as características dos solos determinam similaridades na composição de espécies e na estrutura da vegetação dos campos limpos, foram levantadas as seguintes questões: 1) existe associação da vegetação com as características edáficas? 2) os fragmentos de campo limpos úmidos na área de estudo são similares na composição e estrutura da vegetação?

\section{Material e Métodos}

Área de estudo

O Parque Nacional de Sete Cidades (ParnaSC), criado em 1961, possui uma área de $6.221,48$ ha. Está localizado entre os municípios de Brasileira e Piracuruca $\left(04^{\circ} 02^{\prime}-08^{\prime}\right.$ 'S e $41^{\circ} 40^{\prime}$ $\left.45^{\prime} \mathrm{W}\right)$ no norte do estado do Piaú, com altitudes variando de 100 a $290 \mathrm{~m}$. Segundo a classificação climática de Thornthwaite \& Mather (1955) o clima é considerado subúmido, megatérmico com grande deficiência hídrica no inverno e concentração de $30,3 \%$ da evapotranspiração potencial no trimestre setembro-outubro-novembro (C2w2 A'a'). A precipitação média anual é de $1.650 \mathrm{~mm}$, com $87 \%$ ocorrendo de janeiro a maio.

A vegetação é caracterizada por grande variedade de comunidades, distribuindo-se em mosaicos, incluídas no domínio do Cerrado ou de 
transição Cerrado/Caatinga (IBDF 1979). Oliveira et al. (2007) mapearam a vegetação do Parque e evidenciaram o complexo mosaico de tipos estruturais dominados por formações savânicas (cerrado sentido restrito e cerrado rupestre), além de campo limpo, cerradão, floresta ocasionalmente inundada e estacional semidecídua. A área de ocorrência da vegetação campestre ocupa 14,3\% de extensão total do Parque, principalmente na porção centro-sudoeste, associada, em sua maioria aos Neossolos Quartzarênicos e Planossolos (Oliveira et al. 2007).

\section{Metodologia de amostragem da vegetação}

Através de mapa e imagem de satélite, a área do Parque foi estratificada por fitofisionomias, e seis fragmentos de campo limpo foram selecionados. Os fragmentos têm tamanhos variados (1,2 a 19,2 ha), mas são na maioria pequenos e circundados por matrizes que envolvem duas ou mais fisionomias. Após a estratificação foi adotado o método de interseção na linha proposto por Canfield $(1941,1950) \mathrm{com}$ a instalação de 17 linhas aleatórias de $10 \mathrm{~m}$ cada, distribuídas em seis áreas (Fig. 1), com cinco linhas na primeira, três na segunda e terceira, e duas nas demais, de acordo com o tamanho da área selecionada. Destas, as áreas três e seis permaneciam com lençol freático superficial no período da amostragem. Cada linha foi demarcada e subdividida com varetas de ferro, em segmentos de $1 \mathrm{~m}$, que representaram as subunidades amostrais (UA) para a análise da estrutura da vegetação, perfazendo um total de 170 UA de $1 \mathrm{~m}$ inventariados (Munhoz \& Felfili 2006a). A amostragem foi realizada no final da estação chuvosa/início da seca (julho de 2007).

$\mathrm{O}$ material testemunho foi herborizado e incluído no acervo dos herbários da Universidade Federal do Piauí (TEPB) e Universidade de Brasília (UB). As identificações foram realizadas através de consultas à literatura especializada, por comparação com exemplares nos herbários e, confirmadas por especialistas em grupos taxonômicos. As espécies foram classificadas em famílias de acordo com o sistema do Angiosperm Phylogeny Group III (APG III 2009). Para a família Poaceae foi adotado o Catalogue of the New World Grasses (CNWG) (<http://www. tropicos.org/Project/CNWG $>$ ).

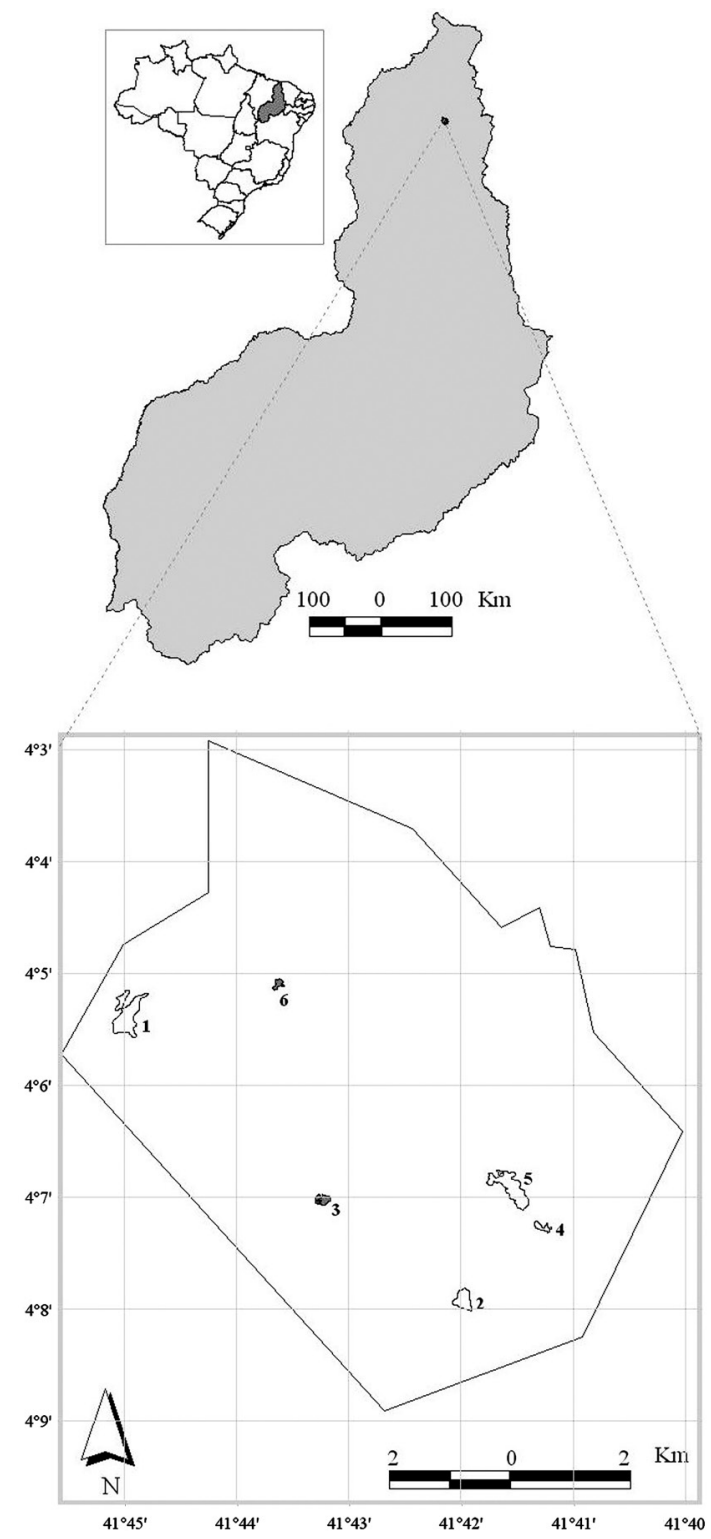

Figura 1 - Localização das áreas de campo limpo amostradas no Parque Nacional de Sete Cidades, Piauí. Área 1 (L1 - L5), Área 2 (L6 - L8), Área 3 (L9 - L11), Área 4 (L12 - L13), Área 5 (L14 - L15), Área 6 (L16 L17). Os campos 3 e 6 estão destacados pois apresentam período maior de alagamento.

Figure 1 - Areas location in moist grassland sites at Sete Cidades National Park, Piauí, Brazil. Site 1 (L1 - L5), Site 2 (L6 - L8), Site 3 (L9 - L11), Site 4 (L12 - L13), Site 5 (L14 - L15), Site 6 (L16 - L17). The sites 3 and 6 were highlighted because show a long period of flooding. 
Coleta das variáveis espaciais e solo

Próxima a região central de cada linha foi coletada uma amostra superficial simples de solo $(0-20 \mathrm{~m} \mathrm{~cm})$. As análises químicas e texturais foram realizadas segundo o protocolo da EMBRAPA (1997). Foram analisadas as seguintes variáveis: $\mathrm{pH}$; teores de $\mathrm{Ca}+\mathrm{Mg}, \mathrm{Ca} / \mathrm{CTC}, \mathrm{Ca} / \mathrm{K}$, $\mathrm{Ca} / \mathrm{MG}, \mathrm{Ca}, \mathrm{Mg}, \mathrm{Al}, \mathrm{H}+\mathrm{Al}, \mathrm{H}+\mathrm{Al} / \mathrm{CTC}, \mathrm{K} / \mathrm{CTC}$, MG/K, K, P, Na, Zn, Cu, Fe e Mn; saturação de bases; saturação de alumínio; matéria orgânica; capacidade de troca catiônica (CTC) e teores de argila, silte e areia.

As variáveis espaciais foram representadas pelas coordenadas binárias da região inicial de cada linha, bem como, de outras sete variáveis derivadas destas, seguindo o modelo sugerido por Borcard $e t$ al. (1992): $\mathrm{x}^{2}, \mathrm{x}^{3}, \mathrm{y}^{2}, \mathrm{y}^{3}, \mathrm{x}^{*} \mathrm{y}, \mathrm{x}^{2 *} \mathrm{y}$ e $\mathrm{x}^{*} \mathrm{y}^{2}$.

\section{Estrutura da vegetação}

Com base na ocorrência e projeção horizontal de cada espécie por UA ao longo das linhas amostradas, foram calculados os seguintes parâmetros fitossociológicos: cobertura absoluta (CA), cobertura relativa (CR), frequência absoluta (FA) e frequência relativa (FR) pelas fórmulas de Kent \& Coker (1992), adaptadas por Munhoz \& Felfili (2006a).

\section{Diversidade, riqueza e similaridade}

Para avaliar a diversidade nas 17 linhas e nas áreas separadamente foi utilizado o índice de Shannon-Wiener na base $e$ (H') adaptado por Munhoz \& Felfili (2006a), utilizando-se em vez de número de indivíduos, os valores de cobertura para espécie como uma medida de abundância. A comparação quanto à significância entre os valores de H' para os pares de áreas inventariadas foi avaliada pelo teste- $t$ de Hutcheson $(\alpha=0,05)$ (Zar 1999), através do programa PAST versão 1.91 (Hammer et al. 2001). O potencial de riqueza entre as áreas foi verificado através da curva espécie $\mathrm{x}$ área e o estimador de riqueza de Jackknife de primeira e segunda ordem (Magurran 1988), calculados pelo Programa PCORD versão 4.2 (McCune \& Mefford 1999). As curvas foram traçadas a partir das médias e intervalos de confiança a $95 \%$ do número cumulativo de espécies (McCune \& Mefford 1999), considerando o metro linear como unidade de medida. A similaridade entre as linhas e entre as áreas foi verificada pelo índice de Sørensen modificado por Chao et al.
(2005), utilizando a cobertura como parâmetro de abundância das espécies nas linhas, a análise foi realizada no programa EstimateS, versão 8.2 (Colwell 2005).

\section{Análises de ordenação}

Com base na cobertura absoluta de todas as espécies nas linhas foi empregado o método de escalonamento multidimensional não métrico (NMDS) (Clarke 1993). Trata-se de uma análise que utiliza como informação a matriz de dissimilaridade entre o conjunto de dados (Kent \& Coker 1992). A escolha do índice de dissimilaridade é crucial e pode determinar diferentes modelos e interpretações dos resultados (Quinn \& Keough 2002). O diagrama foi construído com o índice de Bray-Curtis no PAST versão 1.91 (Hammer et al. 2001).

As relações entre as espécies e as variáveis espaciais e edáficas foram realizadas através de uma análise direta de gradientes, a análise de correspondência canônica (CCA), utilizando o programa CANOCO for Windows versão 4.5 (ter Braak \& Smilauer 1998) e seguindo a rotina proposta por Borcard et al. (1992). Essa abordagem é empregada para discriminar a proporção da variação total dos dados das espécies que é explicada pelas variáveis ambientais e espaciais separadamente, bem como quanto da proporção é explicada pelas duas variáveis juntas e ainda, quanto da variação remanescente não é explicada por nenhumas das duas variáveis (Borcard et al. 1992; Machado et al. 2008). A análise exigiu três matrizes, na primeira matriz foram incluídas 26 espécies com frequência absoluta maior ou igual a $10 \%$. A matriz ambiental foi composta pelos dados químicos e texturais do solo, compondo 26 variáveis, que sofreram padronização pela média e desvio padrão para permitir comparação entre variáveis com unidades diferentes e por isso desuniformes (ter Braak \& Smilauer 1998; Felfili et al. 2007). A última matriz foi elaborada com os dados das coordenadas binárias das linhas e suas derivações: $\mathrm{x}^{2}, \mathrm{x}^{3}, \mathrm{y}^{2}, \mathrm{y}^{3}, \mathrm{x}^{*} \mathrm{y}, \mathrm{x}^{2 *} \mathrm{y}$ e $\mathrm{x}^{*} \mathrm{y}^{2}$.

Foram elaboradas quatro CCAs, onde a primeira $\left(\mathrm{CCA}_{1}\right)$ relacionou a matriz de espécies com as variáveis ambientais. Nesta CCA, foram eliminadas da análise as variáveis que causam multicolinearidade entre os dados e por isso apresentaram alta redundância (fator de inflação da variância $>20$ ) como recomendado por ter Braak \& Smilauer (1998), além das variáveis com baixas 
correlações com os eixos 1 e 2, permanecendo na análise o $\mathrm{Cu}, \mathrm{Fe}$, saturação de alumínio, silte, $\mathrm{Zn}$, saturação de bases e areia. Os gráficos de ordenação das variáveis ambientais, previamente selecionadas, com as linhas de amostragem e espécies foram construídos no programa CANODRAW versão 3.0 (Smilauer 1992). A CCA 2 relacionou a matriz de espécies com as variáveis espaciais, entre as quais, o $\mathrm{x}, \mathrm{x}^{2 *} \mathrm{y}$ e $\mathrm{y}^{3}$ foram selecionados a partir da mesma rotina da $\mathrm{CCA}_{1}$. $\mathrm{A} \mathrm{CCA}_{3}$ relacionou as variáveis ambientais e co-variáveis espaciais e na $\mathrm{CCA}_{4}$ as variáveis espaciais foram relacionadas às co-variáveis ambientais.

A variação na composição e estrutura das espécies nos grupos formados pelas linhas reunidas pelas características edáficas na $\mathrm{CCA}_{1}$ foram testadas pelo procedimento de permutação com múltiplas respostas (MRPP) através do no Programa PCORD versão 4.2 (McCune \& Mefford 1999), utilizando a distância de índice de BrayCurtis como medida de dissimilaridade para a abundância de espécies. MRPP tem a vantagem de não exigir pressupostos (como normalidade multivariada e homogeneidade de variâncias) necessários as análises discriminantes (McCune \& Mefford 1999; Biondini et al. 1988).

\section{Resultados}

Em 76,4\% dos transectos a textura é arenosa, seguida de areia franca $(17,6 \%)$ e franco arenosa $(5,8 \%)$. Os teores de argila e silte somados variaram de 10 a $12 \%$, exceto na L12 na qual a soma dessas partículas foi de $18 \%$ (Tab. 1). As variáveis químicas caracterizaram os solos dos campos limpos estudados como fortemente ácidos e com elevados níveis de alumínio. Cerca de $29 \%$ das linhas amostradas apresentaram solos álicos (saturação por alumínio acima de 50\%). Enquanto, para saturação de bases, todas as linhas são distróficas (abaixo de $50 \%$ ). Considerando os valores registrados para a capacidade de troca catiônica (CTC), aliados aos das variáveis acima, os solos foram caracterizados por apresentarem baixa fertilidade (Tab. 1).

Os maiores coeficientes de variação entre as linhas foram registrados para os valores de $\mathrm{Fe}$, $\mathrm{Zn}, \mathrm{MO}, \mathrm{Al}, \mathrm{Cu}$ e $\mathrm{Mn}$, todos maiores do que $40 \%$. Ao passo que, os nutrientes com menores variações entre as linhas foram $\mathrm{pH}, \mathrm{Ca}, \mathrm{Mg}, \mathrm{H}+\mathrm{Al} / \mathrm{CTC}$ e areia.

\section{Caracterização da vegetação}

Foram amostrados 71 táxons, relacionados a 46 gêneros e 25 famílias na amostra total (Tab. 2). Desses,
67 foram determinados até o nível de espécie, três ao nível de gênero e um trata-se possivelmente de uma nova espécie para a ciência, Mesosetum sp. nov. (Tab. 2). O número de espécies por área variou de 24 a 35 (Tab. 2). Quando considerados os primeiros 20 $\mathrm{m}$ de amostragem a área quatro apresentou o maior potencial de riqueza, seguida da área três com $30 \mathrm{~m}$ amostrados (Fig. 2). Os estimadores de jackknife de $1^{\mathrm{a}}$ e $2^{\mathrm{a}}$ ordem indicaram que a área cinco esteve mais próxima de atingir o seu potencial de riqueza $(27,8 \mathrm{e}$ 28,8 , respectivamente), seguida das áreas dois (40,5 e 46,9), seis (33,7 e 38,2), um (41,9 e 48,6), três (40,8 e 46,9$)$ e quatro $(40,5$ e 46,9$)$.

A diversidade de Shannon variou de acordo com diferenças de riqueza de espécies entre as áreas analisadas separadamente (Apêndice). A área 1 apresentou diferenças significativas na diversidade em relação às demais áreas (área $2: \mathrm{t}$ $=3,92, p<0,01$; área 3: $\mathrm{t}=2,07, p<0,05$; área 4: $\mathrm{t}=2,72, p<0,05$; área $5: \mathrm{t}=3,28, p<0,01$; área $6: \mathrm{t}=2,97, p<0,01)$. O valor de $H^{\prime}$ da área 2 foi significativamente menor do que na área $3(\mathrm{t}=$ $-2,80, p<0,01)$, enquanto a diversidade da área 3 foi significativamente maior do que a cinco $(\mathrm{t}=$ $-2,80, p<0,05)$. As demais comparações entre as áreas não diferiram significativamente.

As similaridades pelo índice de ChaoSørensen variaram de 0,70 a 0,87 entre as áreas que não permaneciam com lençol freático superficial durante a amostragem (áreas 1, 2, 4 e 5) e entre as áreas 3 e 6 que permaneciam. As menores similaridades foram registradas entre as áreas 6 e 2 (Chao-Sørensen $=0,11$ ), seis e cinco (ChaoSørensen $=0,15)$, seis e quatro (Chao-Sørensen $=$ $0,17)$ e três e quatro (Chao-Sørensen $=0,22)$. Em

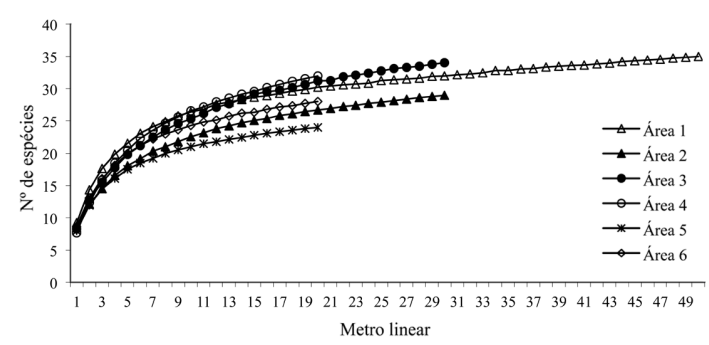

Figura 2 - Curvas de progressão espécie x área com o incremento de unidades amostrais (metro linear), para as seis áreas de campo limpo amostradas no Parque Nacional de Sete Cidades, Piauí.

Figure 2 - Species $x$ area progression curves with the increasing number of sample units (linear meter) for the six moist grassland sites in the Sete Cidades National Park, Piauí, Brazil. 


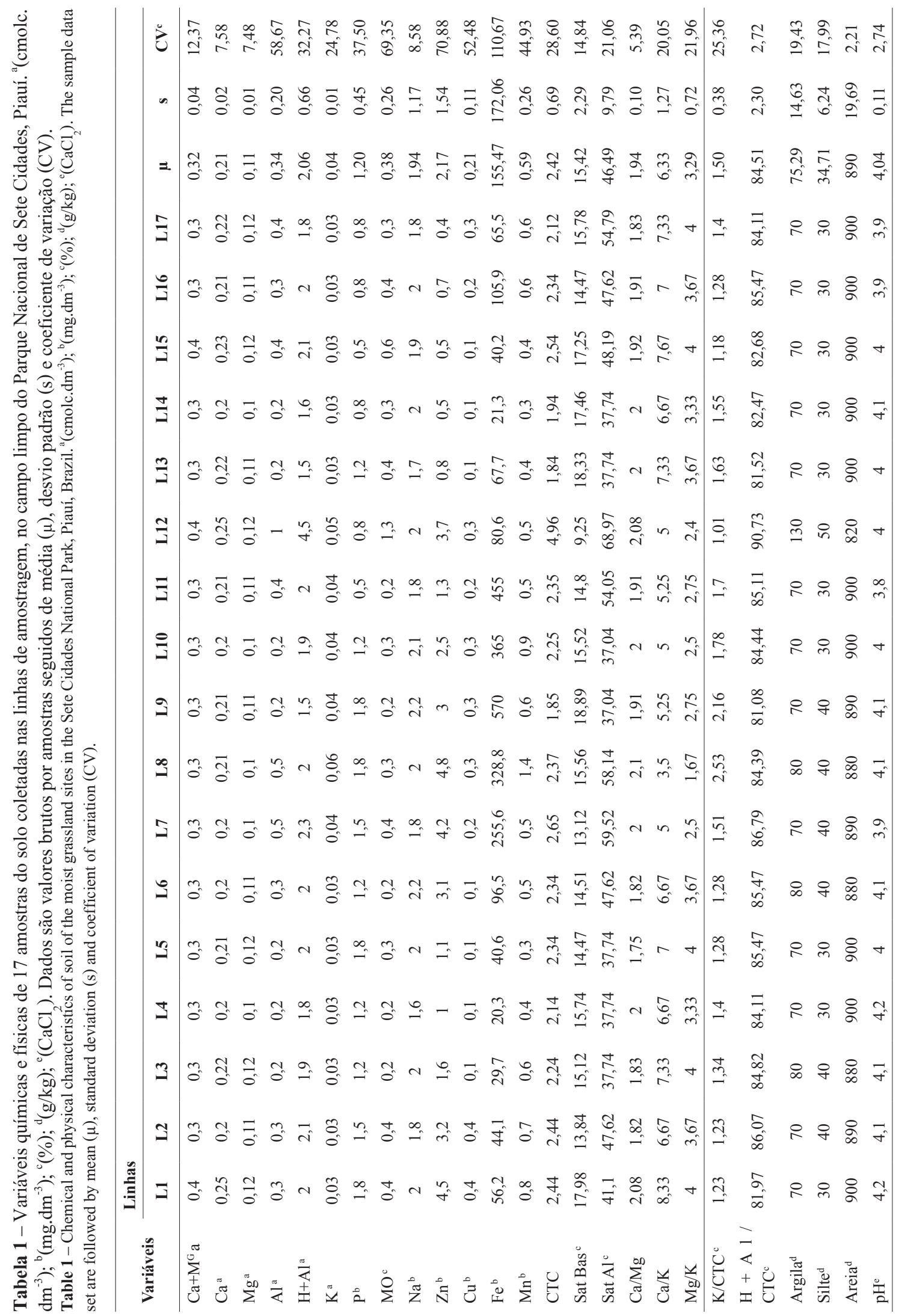


geral, as similaridades entre as linhas da mesma área foram maiores do que entre áreas diferentes pela dependência espacial dos dados (Tab. 2). A NMDS refletiu as diferenças de similaridades entre as áreas (Fig. 3).

Houve modificações entre as espécies com maiores coberturas relativas entre as áreas, Trachypogon spicatus (L.) Kuntze destacou-se nas áreas 1, 2, 4 e 5, enquanto nas áreas 3 e 6 destacaram-se Rhynchospora barbata (Vahl) Kunth e Rhynchanthera grandiflora (Aubl.) DC., respectivamente (Tab. 2).

A distância espacial entre as linhas foi responsável por apenas $16,8 \%$ da variação das espécies. Sete variáveis ambientais relacionadas na $\mathrm{CCA}_{1}(\mathrm{Cu}, \mathrm{Fe}$, saturação de alumínio, silte, $\mathrm{Zn}$, saturação de bases e areia) foram responsáveis por $37,9 \%$ da variação explicada, enquanto a combinação de distância e variáveis ambientais explicou 24,3\%. Restaram $21 \%$ da variação que permaneceu não explicada, ou seja, representada de forma estocástica ou por variáveis não mensuradas neste trabalho.

Todas as CCAs apresentaram gradientes curtos, mas os autovalores foram significativos para o primeiro eixo nas duas primeiras $(>0,3)$. As correlações de Pearson entre as espécies e as variáveis testadas nas quatro CCAs foram elevadas, enquanto os testes de Monte Carlo indicaram diferenças significativas entre as correlações geradas pelo acaso e a real nas duas primeiras CCAs para o primeiro e segundo eixos canônicos. Considerando a $\mathrm{CCA}_{1}$, a partir daqui escolhida para representar a análise, as variáveis mais correlacionadas com os dois primeiros eixos canônicos foram a saturação

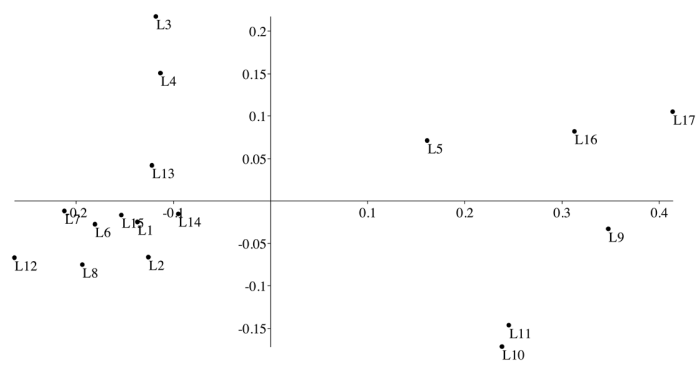

Figura 3 - Diagrama de ordenação pelo método de escalonamento multidimensional não métrico (NMDS) das 17 linhas amostradas nas áreas de campo limpo no Parque Nacional de Sete Cidades, Piauí, com base na cobertura absoluta das 71 espécies (Stress $=0,11$ ). Figura 3 - Non-metric multi-dimensional scaling (MDS) of the 17 lines sampled in the grassland sites in the Sete Cidades National Park, Piauí, based on the absolute cover of 71 species (Stress $=0,11$ ). de alumínio com o segundo eixo, e zinco e silte com o primeiro, todas com correlação positiva. $\mathrm{Na}$ $\mathrm{CCA}_{2}$ as variáveis espaciais $\mathrm{x}$ e $\mathrm{x}^{2 *} \mathrm{y}$ apresentaram correlações positivas com o segundo e primeiro eixo, respectivamente (Tab. 3).

O diagrama de ordenação da CCA $_{1}$ (Fig. 4a) separou três grupos, de acordo com suas variações edáficas. O primeiro formado pelas linhas L1, L3, L4, L5, L13 e L14, que embora posicionadas em quadrantes diferentes, foram correlacionadas a valores elevados de saturação de bases e solos mais drenados. O segundo formado pelas linhas L6, L7, L8, L12 e L15 que apresentaram maiores teores de zinco, silte e saturação de alumínio. O terceiro, correlacionado com $\mathrm{Fe}$ e $\mathrm{Cu}$ que reuniu as linhas das áreas 3 e 6 (L9, L10, L11, L16 e L17) e a L2. Os grupos propostos pela $\mathrm{CCA}_{1}$ apresentaram diferenças significativas na $\operatorname{MRPP}(\mathrm{A}=0,19, p<0,01)$.

No primeiro grupo, houve um conjunto de espécies mais frequentes em solos com o aumento da fertilidade, como por exemplo, Pterolepis polygonoides (DC.) Triana e Syngonanthus sp., e outro conjunto, como Xyris paraensis Poeppig ex Kunth e Drosera sessilifolia A. St.-Hil mais frequentes em solos mais arenosos. Nas linhas menos férteis, as espécies mais relacionadas ao segundo grupo foram Acisanthera bivalvis (Aubl.) Cogn, Elephantopus hirtiflorus DC., T. spicatus, Scleria leptostachya Kunth e Croton pedicellatus Kunth. Enquanto, Paepalanthus minutulus Mart., Chamaecrista desvauxii var. chapadicola H.S.Irwin \& Barneby estiveram mais relacionadas com os teores de Fe e Cu no último grupo (Fig. 4b).

\section{Discussão}

A elevada acidez encontrada nas áreas de campo limpo do ParnaSC é comum a outras fisionomias do Parque (Oliveira 2004; Lindoso 2008; Moura 2010). No geral, os solos dos campos foram semelhantes aos padrões do Cerrado, como baixos valores de $\mathrm{pH}$, cálcio, magnésio, fósforo e altos teores de alumínio e ferro (Furley \& Ratter 1988), mas apresentaram menor fertilidade quando comparados com áreas de campos do Brasil Central (Amorim \& Batalha 2007; Munhoz et al. 2008). Isto pode estar associado ao material de origem dos solos do Piauí, formados por rochas da Bacia Sedimentar do Parnaíba ou da Província Sedimentar do Meio-Norte, enquanto que os solos do Planalto Central são mais antigos, formados por rochas do Pré-Cambriano até o presente, apresentando rochas magmáticas, metamórficas e sedimentares (Ab’Saber 2003; Barros \& Castro 2006). 


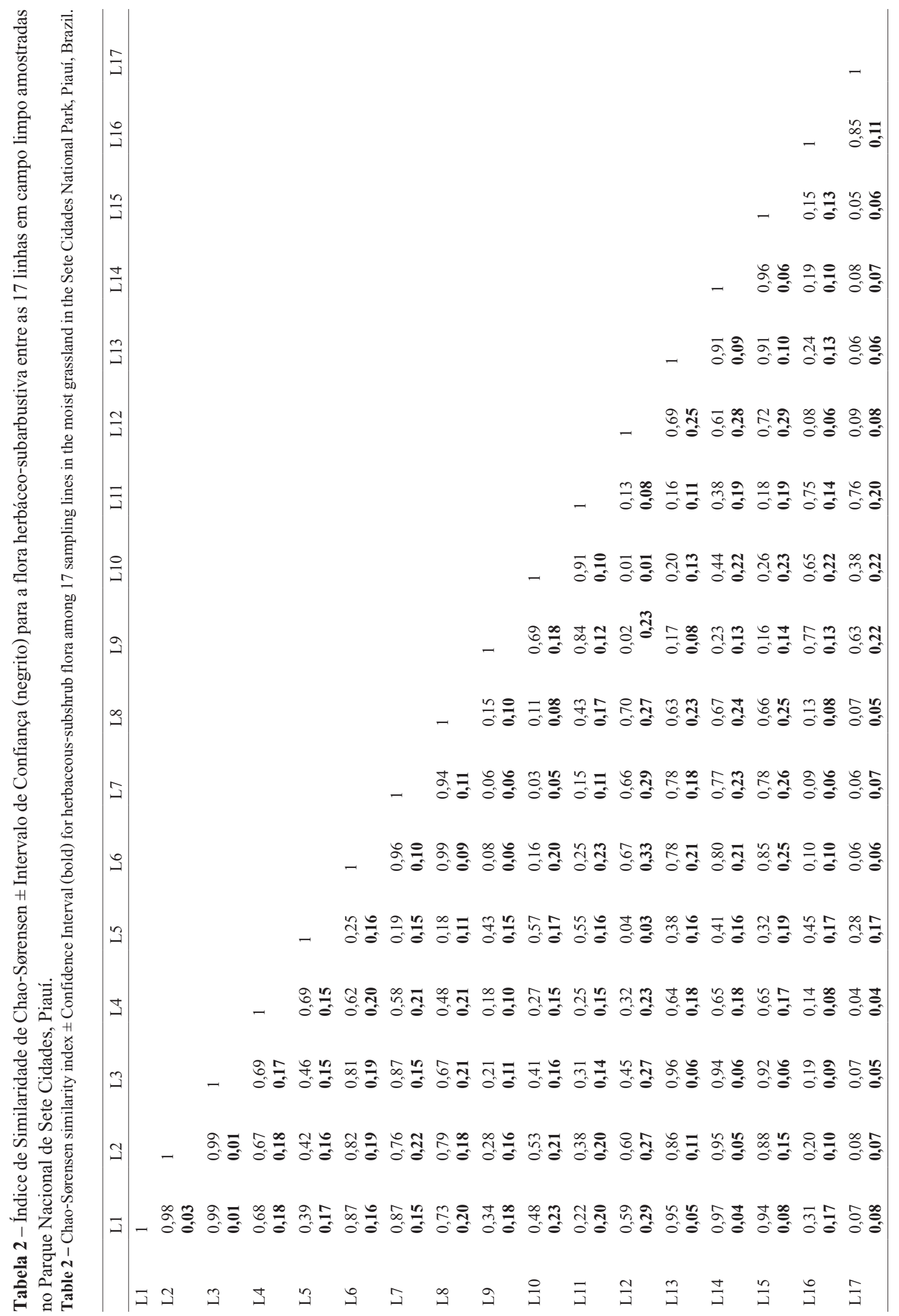


Relação entre a vegetação e as propriedades do solo





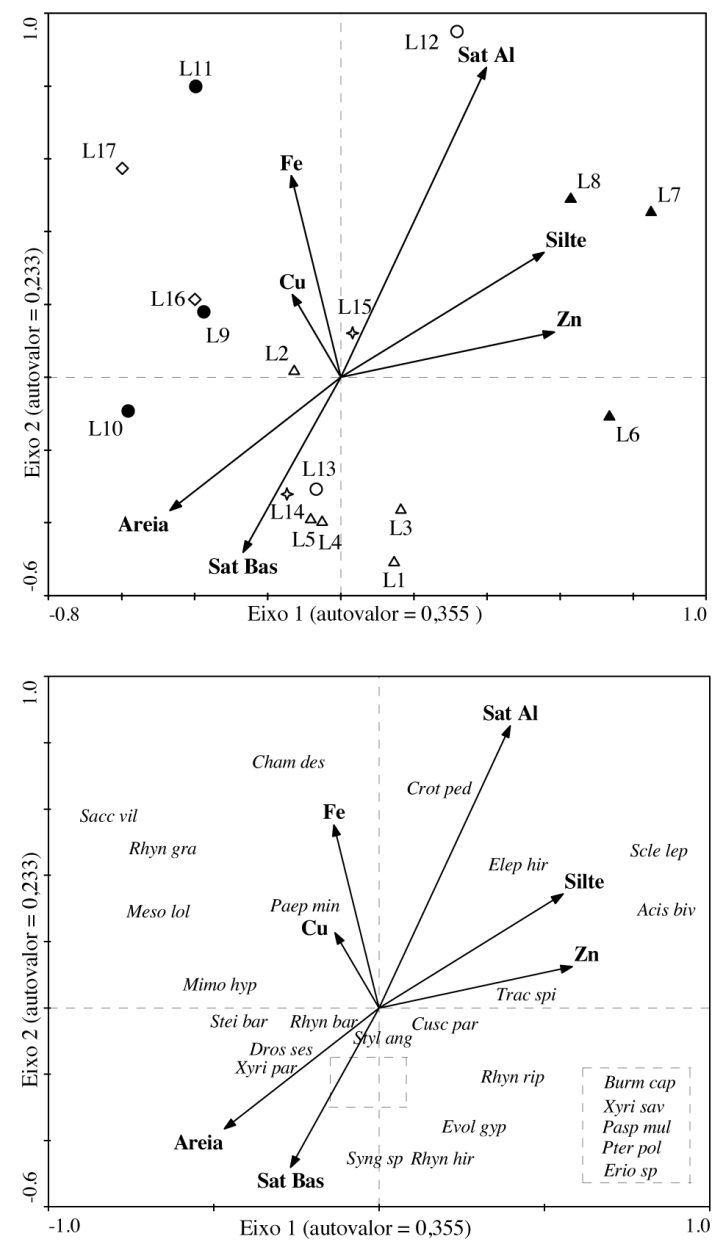

Figura 4 - Análise de correspondência canônica (CCA1). A. Diagrama de ordenação das 17 linhas e das variáveis ambientais nos dois primeiros eixos, com base nos dados de frequência absoluta das 26 espécies $(\geq 10 \%$ ). B. Diagrama de ordenação das espécies e das variáveis ambientais nos dois primeiros eixos. As espécies estão identificadas pelas primeiras letras do binômio, ver Tabela 2 .

Figure 4 - Canonical correspondence analysis (CCA1). AOrdination diagrams for 17 lines and environmental variables in first two axes, based on absolute frequency data of 26 species $(\geq 10 \%)$. B- Ordination diagrams for species and environmental characteristics in first two axes. Species were identified by the first two letters (see Table 2).

As diferenças no teor de alguns elementos entre as seis áreas, como a saturação de alumínio e de bases, além daqueles que apresentaram maior coeficiente de variação para as 17 linhas conjuntamente, evidencia a heterogeneidade edáfica entre alguns campos do Parque. As áreas 3 e 4, que apresentaram maiores teores de ferro e alumínio, respectivamente, registraram maior potencial de riqueza de espécies. Correlação direta entre a limitação de diversos nutrientes e o aumento na riqueza de espécies foi encontrada em campos temperados (Janssens et al. 1998). Amorim \& Batalha (2008) também sugerem que o excesso de alumínio limita o fornecimento de nutrientes, ocorrendo uma correlação positiva com o aumento do número de espécies no Parque Nacional das Emas.

A variação no número de espécies entre as linhas não se deu em função da variação da umidade superficial entre as mesmas, observada no momento da amostragem nas áreas, um padrão comum para outras áreas campestres, onde o aumento da umidade determinou diminuição da riqueza (Goldsmith 1974; Guimarães et al. 2002; Munhoz \& Felfili 2008). Por outro lado, houve baixa similaridade entre as linhas das áreas mais secas e mais úmidas, evidenciando modificações na composição das espécies e na estrutura da vegetação, apesar do intervalo de confiança significativo para poucas comparações. Possivelmente, as diferenças na duração de alagamento entre as áreas funcionaram como um filtro selecionando espécies capazes de suportar tal condição (Chase 2003).

Os seis campos estudados apresentaram poucas espécies respondendo pelas maiores coberturas relativas. No geral, a dominância ecológica de algumas espécies é influenciada por condições ambientais extremas, como a baixa disponibilidade de nutrientes no solo e estresse hídrico causado pelo excesso ou falta de água (Ashton 1990). Para o estrato arbóreo do Cerrado, parte do sucesso para a dominância de espécies se deve a mecanismos adaptativos, como capacidade de acúmulo de alumínio em seus tecidos e diminuição da exigência nutritiva (Franco 2005; Haridasan 2008). Para o estrato herbáceo, a exigência é extremamente baixa, um aspecto importante na manutenção de processos que garantam a resiliência em ambiente savânico (Villela \& Haridasan 1994; Haridasan 2008).

As famílias que se destacaram no número de espécies foram, em geral, bem representadas em trabalhos que investigaram o estrato herbáceo. Cyperaceae apresentou o maior número de espécies, com destaque para gênero Rhynchospora. Essa família, associada à Xyridaceae e Eriocaulaceae, ocorre com frequência em áreas de campo limpo úmido e veredas (Guimarães et al. 2002; Munhoz \& 
Felfili 2006b, 2007). O registro proporcionalmente elevado de espécies de Fabaceae, o que não é comum no campo úmido por se tratar de um ambiente com teores mais elevados de fósforo (Amorim \& Batalha 2007), pode estar relacionado à menor fertilidade da área de estudo.

No campo úmido, a matéria orgânica é uma das propriedades do solo correlacionada com a determinação da estrutura da vegetação (Amorim \& Batalha 2007; Munhoz et al. 2008), possivelmente pela condição de alagamento dessas áreas ditarem modificações no solo interferindo nos processos de decomposição (Gopal \& Masing 1990), aumentando a quantidade de matéria orgânica no solo (Crawley 1997). No entanto, esta não foi uma variável selecionada na análise de ordenação das áreas de campo limpo úmido do ParnaSC. A localização do Parque em uma zona de ecótonos, conhecida como área de tensão ecológica (Rivas 1996), bem como sua posição limítrofe no bioma Cerrado são determinantes do ambiente. Segundo Castro \& Martins (1999), os cerrados do Nordeste são delimitados pelo Polígono das Secas, com condições climáticas diferentes das encontradas no Planalto Central. Dessa forma, os campos limpos úmidos estudados não estão sujeitos a alagamento prolongado, com poucas áreas passando por um período maior de alagamento, limitado principalmente aos quatro meses de maior pluviosidade na região.

As áreas 3 e 6, similares entre si, apresentavam lençol freático superficial em julho, período que marca o início da estação seca. As espécies com maiores coberturas nessas áreas não coincidiram com as outras áreas mais secas amostradas, sendo, portanto, caracterizados dois subtipos de comunidades de campo úmido no Parque com base no período de alagamento, com separação evidente na análise NMDS. Segundo Critchey et al. (2002), a quantidade de alagamento e a duração do período de inundação são fatores importantes para diferenciar comunidades de campos úmidos.

A alta correlação entre espécies e ambiente mostrou forte associação entre a vegetação e as características edáficas. Dois gradientes foram selecionados na análise CCA: um de textura e o outro de fertilidade dos solos, separando as linhas amostradas em três grupos em consequência da frequência das espécies mais comuns. No primeiro grupo, foi possível separar linhas relacionadas à areia e saturação de bases, representada principalmente pelas áreas 1 e 5 .
Outro grupo esteve mais associado ao zinco, saturação de alumínio e silte, neste caso reunindo a área 2, além da linha L12. O último grupo reuniu as áreas 3 e 6, além da linha L2, mais associadas ao cobre e ferro. A confirmação dos grupos foi reforçada pelas diferenças significativas indicadas na MRPP. Mesmo com baixa fertilidade nos solos em todas as áreas, as variações entre elas foram refletidas nas diferenças de composição e estrutura da vegetação de campo limpo no ParnaSC. Por outro lado, a posição das linhas L2, L15 e L12 sugere a heterogeneidade edáfica dentro de algumas áreas.

Análises de ordenação, como a realizada neste trabalho, mostram tendências da distribuição da vegetação, mas nem sempre revelam relações diretas com as variáveis testadas. Com a inclusão da variável espacial na análise foi possível mensurar o quanto da variação total dos dados foi explicada pelo espaço. As variáveis selecionadas $\mathrm{x}, \mathrm{x}^{2 *} \mathrm{y}$ e $y^{3}$ corresponderam ao eixo de maior variação das linhas no campo, ou seja, leste-oeste. Parte da variação permaneceu não explicada, mas, de acordo com ter Braak (1988), mesmo valores baixos podem conter informações significativas e importantes na interpretação da distribuição das espécies e na sua relação com o ambiente.

Portanto, com base nos resultados encontrados no presente estudo, pode-se sugerir que existe associação da vegetação com as características edáficas no ParnaSC, os solos das seis áreas embora com baixa fertilidade e pH ácido, apresentaram diferenças nos teores de alguns elementos e na textura que ditaram modificações na composição e estrutura das espécies. Tais características aliadas as diferenças na umidade superficial entre as áreas formaram dois grupos com baixa similaridade entre si, o primeiro reuniu as áreas mais secas e o segundo as mais úmidas.

A escassez de unidades de conservação na porção norte do Estado do Piauí, aliada a ausência de gramíneas exóticas invasoras nas áreas de campo limpo estudadas no PNSC, reforça a idéia que esses ambientes devem ser preservados e inventariados do ponto de vista da estrutura, dinâmica e relação com as variáveis ambientais, buscando delinear estratégias de conservação da sua diversidade biológica.

\section{Agradecimentos}

À Profa. Jeanine Maria Felfili (in memoriam) a valiosa ajuda no início deste trabalho e o exemplo de profissional que deixou para todos a sua volta; 
aos funcionários do ParnaSC, Superintendência Estadual do IBAMA e Instituto Chico Mendes em Teresina/PI; à Jéssica Viana e Rosemary Sousa a contribuição nos trabalhos de campo; à Fundação de Amparo à Pesquisa do Estado do Piauí (FAPEPI) a bolsa concedida para a primeira autora; ao PELD site 10; aos especialistas das diversas famílias que contribuíram na identificação do material botânico.

\section{Referências}

Ab'Saber, A.N. 2003. Os domínios de natureza no Brasil: potencialidades paisagísticas. Ateliê Editorial, São Paulo. 160p.

Al-Mufti, M.M.; Sydes, C.L.; Furness, S.B.; Grime, J.P. \& Band, S.R. 1977. A quantitative analysis of shoot phenology and dominance in herbaceous vegetation. Journal of Ecology 65: 759-791.

Alvim, P.T. \& Araújo, W.A. 1952. El suelo como factor ecológico en el desarollo de la vegetación en el centro-oeste del Brasil. Turrialba 2: 153-160.

Amorim, P.K. \& Batalha, M.A. 2006. Soil characteristic of a hyperseasonal cerrado compared to a seasonal cerrado and a floodplain grassland: implications for plant community structure. Brazilian Journal of Biology 66: 661-670.

Amorim, P.K. \& Batalha, M.A. 2007. Soil-vegetation relationships in hyperseasonal cerrado, seasonal cerrado, and wet grassland in Emas National Park (central Brazil). Acta Ecologica 32: 319-327.

Amorim, P.K. \& Batalha, M.A. 2008. Soil chemical factors and grassland species density in Emas National Park (central Brazil). Brazilian Journal of Biology 68: 279-285.

Angiosperm Phylogeny Group (APG III). 2009. An update of the Angiosperm Phylogeny Group classification for the orders and families of flowering plants: APG III. Botanical Journal of the Linnean Society 161: 105-121.

Araújo, G.M.; Barbosa, A.A.A.; Arantes, A.A. \& Amaral, A.F. 2002. Composição florística de veredas do município de Uberlândia. Revista Brasileira de Botânica 25: 475-493.

Arens, K. 1958. O cerrado como vegetação oligotrófica. Boletim da Faculdade de Filosofia, Ciências e Letras da Universidade de São Paulo, Botânica 15: 59-77.

Ashton, P.S. 1990. Species richness in tropical forests. In: Holm-Nielsen, L.B.; Nielsen, I.C. \& Balslev, H. (eds.). Tropical forests - botanical dynamics, speciation and diversity. Academic Press, London. Pp. 239-251.

Barros, J.S. \& Castro, A.A.J.F. 2006. Compartimentação geoambiental no complexo de Campo Maior, PI: uma área de tensão ecológica. Interações 8: 119-130.

Biondini, M.E.; Mielke, P.W. \& Berry, K.J. 1988. Data dependent permutation techniques for the analysis of ecological data. Vegetatio 75: 161-168.
Borcard, D.; Legendre, P. \& Drapeau, P. 1992. Partialling out the spatial component of ecological variation. Ecology 73: 1042-1055.

Canfield, R. 1941. Application of line interception in sampling range vegetation. Journal of Forestry 39 : 388-394.

Canfield, R. 1950 (reprinted Sept.). Sampling range by the line interception method. Research Report 4. Southwestern Forest and Range Experiment Station. 28p.

Castro, A.A.J.F.; Martins, F.R.; Tamashiro, J.Y. \& Shepherd, G.J. 1999. How rich is the woody flora of Brazilian Cerrados? Annals of the Missouri Botanical Garden 86: 192-224.

Catalogue of the New World Grasses (CNWG). Disponível em <http://mobot.mobot.org/W3T/Search/nwgc. html >. Acesso em 5 Jan 2011.

Chao, A.; Chazdon, R.L.; Colwell, R.K. \& Shein, T.J. 2005. A new statistical approach for assessing similarity of species composition with incidence and abundance data. Ecology Letters 8: 148-159.

Chase, J.M., 2003. Community assembly: when should history matter? Oecologia 136: 489-498.

Colwell, R.K. 2005. EstimateS: Statistic estimation of species richness and shared species from samples. Version 8.2. Disponível em $<$ http://viceroy.eeb. uconn.edu/estimates $>$. Acesso em 15 Set 2009.

Crawley, M.J. 1997. The structure of plant communities. In: Crawley, M.J. Plant ecology. $2^{\text {nd }}$ ed. Blackwell, Oxford. Pp. 475-531.

Critchley, C.N.R.; Chambers, B.J.; Fowbert, J.A.; Sanderson, R.A., Bhogal, A. \& Rose, S.C. 2002. Association between lowland grassland plant communities and soil properties. Biological Conservation 105: 199-215.

Empresa Brasileira de Pesquisa Agropecuária. 1997. Manual de Método de Análise de Solo, 2ed. Centro Nacional de Pesquisa de Solos, Rio de Janeiro. 212p.

Felfili, J.M.; Carvalho, F.A.; Líbano, A.M.; Venturoli, F.\& Pereira, B.A.S. 2007. Análise multivariada em estudos da vegetação. Universidade de Brasília, Brasília. 60p.

Franco, A.C. 2005. Biodiversidade de forma e função: implicações ecofisiológicas das estratégias de utilização de água e luz em plantas lenhosas do cerrado. In: Scariot. A.; Felfili, J.M. \& SousaSilva, J.C. (eds.). Cerrado: ecologia, biodiversidade e conservação. Ministério de Meio Ambiente, Brasília. Pp. 179-196.

Furley, P.A. \& Ratter, J.A. 1988. Soil resources and plant communities of the Central Brazilian cerrado and their development. Journal of Biogeography 15: $97: 108$.

Goldsmith, F.B. 1974. Multivariate analyses of tropical grassland communities in Mato Grosso, Brasil. Journal of Biogeography 1: 111-122. 
Gopal, B. \& Masing, V. 1990. Biology and ecology. In: Patten, B.C. (ed.). Wetlands and shallow continental water bodies. SPB Academic Publishing, The Hague. Pp. 91-239.

Grime, J.P. 1979. Plant strategies and vegetation processes. John Wiley \& Sons, Chichester. 186p.

Guimarães, A.J.M.; Araújo, G.M.; Oliveira, A.A. \& Corrêa, G.F. 2002. Estrutura fitossociológica de uma área natural e antropizada de uma vereda de Uberlândia, MG. Acta Botanica Brasilica 16: 317-329.

Haridasan, M., 2000, Nutrição mineral de plantas nativas do cerrado. Revista Brasileira de Fisiologia Vegetal 12: 54-64.

Hammer, Ø.; Harper, D.A.T. \& Ryan, P.D. 2001. PAST: Paleontological Statistics Software Package for Education and Data Analysis. Palaeontologia Electronica 4: 9.

Haridasan, M. 2008. Nutritional adaptations of native plants of the cerrado biome in acid soils. Brazilian Journal of Plant Physiology 20: 183-195.

Instituto Brasileiro de Desenvolvimento Florestal (IBDF). 1979. Plano de Manejo: Parque Nacional de Sete Cidades, M.A/Fundação Brasileira para a Conservação da Natureza (FBCN), Brasília. 61p.

Janssens, F.; Peeters, A.; Tallowin, J.R.B.; Bakker, J.P.; Bekker, R.M.; Fillat, F. \& Oomes, M.J.M. 1988. Relationship between soil chemical factors and grassland diversity. Plant and Soil 202: 69-78.

Kent, M. \& Coker, P. 1992. Vegetation description and analysis; a pratical Approach. Belhaven Press, London. 363p.

Lindoso, G.L. 2008. Cerrado sensu stricto sobre Neossolo Quartzarênico: fitogeografia e conservação. Dissertação de Mestrado. Universidade de Brasília, Brasília. 170p.

Machado, E.L.M.; Oliveira-Filho, A.T.; van der Berg, E.; Carvalho, W.A.C.; Souza, J.S.; Marques, J.J.G.S.M. \& Calegário, N. 2008. Efeito do substrato, bordas e proximidade espacial na estrutura de comunidade arbórea de um fragmento florestal em Lavras, MG. Revista Brasileira de Botânica 31: 287-302.

Magurran, A.E. 1988. Ecological Diversity and its Measurement. Croom Helm, London. 178p.

McCune, B. \& Mefford, M.J. 1999. PC-ORD version 4.2, multivariate analysis of ecological data, Users guide. MiM Software Design, Glaneden Beach, Oregon.

Medina, E. 1996. Biodiversity and nutrient relations in savanna ecosystems: Interation between primary producers, soil microorganisms, and soils. In: Solbrig, O.T.; Medina, E. \& Silva, J.F. (eds.). Biodiversity and savanna ecosystem processes. Elsevier, Berlin. Pp.45-55.

Montgomery, R.F. \& Askew, G.P. 1983. Soils of tropical savannas. In: Bourlière, F. Ecosystems of the world - tropical savannas. Elsevier, Berlin. Pp.63-78.
Moreno, M.I.C.; Schiavini, I. \& Haridasan, M. 2007. Fatores edáficos influenciando na estrutura de fitofisionomias do cerrado. Caminhos da Geografia 9: 173-194.

Moura, I.O. 2010. Fitogeografia do cerrado rupestre: relações florísticas-estruturais e ecológicas de espécies lenhosas. Tese de Doutorado. Universidade de Brasília, Brasília. 247p.

Munhoz, C.B.R. \& Felfili, J.M. 2006a. Fitossociologia do estrato herbáceo-subarbustivo de uma área de campo sujo no Distrito Federal, Brasil. Acta Botanica Brasílica 20: 671-685.

Munhoz, C.B.R. \& Felfili, J.M. 2006b. Floristics of the herbaceous and subshrub layer of a moist grassland in the cerrado biosphere reserve (Alto Paraíso de Goiás), Brazil. Edinburgh Journal of Botany 63: 343-354.

Munhoz, C.B.R. \& Felfili, J.M. 2007. Florística do estrato herbáceo-subarbustivo de um campo limpo úmido em Brasília, Brasil. Biota Neotropica 7: 205-215.

Munhoz, C.B.R.; Felfili, J.M. \& Rodrigues, C. 2008. Species-environment relationship in the herb-subshrub layer of a moist Savanna site, Federal District, Brazil. Brazilian Journal of Biology 68: 25-35.

Munhoz C.B.R.; Felfili J.M. 2008. Fitossociologia do estrato herbáceo-subarbustivo em campo limpo úmido no Brasil Central. Acta Botanica Brasilica 22: 905-913.

Oliveira, M.E.A. 2004. Mapeamento, florística e estrutura da transição campo-floresta na vegetação (cerrado) do Parque Nacional de Sete Cidades, nordeste do Brasil. Tese de Doutorado. Universidade Estadual de Campinas, Campinas. 151p.

Oliveira, M.E.A.; Martins, F.R.; Castro, A.A.J.F. \& Santos, J.R. 2007. Classes de cobertura vegetal do Parque Nacional de Sete Cidades (transição campo-floresta) utilizando imagens TM/Landsat, NE do Brasil. In: XIII Simpósio Brasileiro de Sensoriamento Remoto, 2007, Florianópolis. Anais (Proceedings) do XIII SBSR. Vol. 13. Pp. 1775-1783.

Quinn, G.P. \& Keough, M.J. 2002. Experimental design and data analysis for biologists. Cambridge University Press, New York. 537p.

Ramos, M.V.V.; Curi, N.; Motta, P.E.F.; Vitorino, A.C.T.; Ferreira, M.M. \& Silva, M.L.N. 2006. Veredas do triângulo mineiro: solos, água e uso. Ciência e Agrotecnologia 30: 283-293.

Rivas, M.P. 1996. Macrozoneamento geoambiental da bacia hidrográfica do rio Parnaíba. IBGE, Rio de Janeiro. Série Estudos e Pesquisas em Geociências, 4. 110p

Ruggiero, P.G.C.; Batalha, M.A.; Pivello, V.R. \& Meirelles, S.T. 2002. Soil-vegetation relationships in cerrado (Brazilian savanna) and semideciduous forest, Southeastern Brazil. Plant Ecology 160: 1-16. 
Smilauer, P. 1992. CANODRAW: user's guide. Version 3.0. Microcomputer Power. Ithaca, New York. $118 \mathrm{p}$.

Tannus, J.L.S. \& Assis, M.A. 2004. Composição de espécies vasculares de campo sujo e campo úmido em área de cerrado, Itirapina - SP, Brasil. Revista Brasileira de Botânica 27: 489-506.

ter Braak, C.J.F. 1988. CANOCO - A FORTRAN program for canonical community ordination by (Partial) (Detrended) (Canonical) correspondence analysis and redundancy analysis, version 2.1. Technical report LWA-88-2, TNO, Wageningen, Intitute of Applied Computer Science. 95p. ter Braak, C.J.F. \& Smilauer, P. 1998. CANOCO Reference manual and user's guide to Canoco for Windows: software for canonical community ordination, version 4.5 Microcomputer Power. Ithaca, New York. 351p.

Thornthwaite, C.W. \& Mather, J.R. 1955. The water balance. Publications in Climatology, 8. Drexel Institute of Technology, Centerton, New Jersey. 104p.

Villela, D.M.V.; Haridasan, M. 1994. Response of the ground layer community of a cerrado vegetation in central Brazil to liming and irrigation. Plant and Soil 163:25-31.

Zar, J. H. 1999. Biostatistical analysis. 4ed. PrenticeHall, New Jersey. 662p. 


\section{Relação entre a vegetação e as propriedades do solo em áreas de campo limpo úmido no Parque Nacional de Sete Cidades, Piauí, Brasil}

Vegetation and soil relationship in moist grassland

in the National Park of Sete Cidades, Piauí, Brazil

Maura Rejane de Araújo Mendes, Cássia Beatriz Rodrigues Munhoz,

Manoel Cláudio da Silva Júnior \& Antonio Alberto Jorge Farias Castro

Apêndice - Cobertura relativa e frequência relativa (negrito) das espécies de 17 linhas de amostragem do estrato herbáceo-subarbustivo de campo limpo do Parque Nacional de Sete Cidades, Piauí.

Appendix - Relative cover and Relative frequency (bold) of the species in 17 sampling lines of the herbaceous-subshrub layer of the moist grassland sites in the Sete Cidades National Park, Piauí, Brazil.

\begin{tabular}{|c|c|c|c|c|c|c|c|c|c|c|c|c|c|c|c|c|c|}
\hline \multirow[t]{2}{*}{ Família/espécie } & \multicolumn{5}{|c|}{$\begin{array}{l}\text { C. limpo } 1 \\
\text { (L1-L5) }\end{array}$} & \multicolumn{3}{|c|}{$\begin{array}{l}\text { C. limpo } 2 \\
\text { (L6-L8) }\end{array}$} & \multicolumn{3}{|c|}{$\begin{array}{l}\text { C. limpo } 3 \\
\text { (L9-L11) }\end{array}$} & \multicolumn{2}{|c|}{$\begin{array}{l}\text { C. limpo } 4 \\
\text { (L12-L13) }\end{array}$} & \multicolumn{2}{|c|}{$\begin{array}{l}\text { C. limpo } 5 \\
\text { (L14-L15) }\end{array}$} & \multicolumn{2}{|c|}{$\begin{array}{l}\text { C. limpo } 6 \\
\text { (L16-L17) }\end{array}$} \\
\hline & L1 & $\mathbf{L 2}$ & L3 & L4 & $\mathbf{L 5}$ & L6 & L7 & L8 & L9 & L10 & L11 & L12 & L13 & L14 & L15 & L16 & $\mathbf{L 1 7}$ \\
\hline ASTERACEAE & & & & & & & & & & & & & & & & & \\
\hline $\begin{array}{l}\text { Aspilia attenuata (Gardn.) } \\
\text { Baker }\end{array}$ & - & - & - & - & - & - & - & - & - & - & $\begin{array}{l}5,00 \\
2,38\end{array}$ & - & - & - & - & - & - \\
\hline $\begin{array}{l}\text { Elephantopus hirtiflorus } \\
\text { DC. } \\
\text { BURMANNIACEAE }\end{array}$ & $\begin{array}{l}0,22 \\
\mathbf{0 , 9 3}\end{array}$ & $\begin{array}{l}4,31 \\
\mathbf{5 , 8 8}\end{array}$ & $\begin{array}{l}0,27 \\
\mathbf{2 , 0 0}\end{array}$ & - & $\begin{array}{l}0,26 \\
\mathbf{1 , 3 0}\end{array}$ & $\begin{array}{l}1,37 \\
\mathbf{6 , 4 1}\end{array}$ & $\begin{array}{c}7,32 \\
10,71\end{array}$ & $\begin{array}{c}5,90 \\
10,75\end{array}$ & $\begin{array}{l}2,04 \\
\mathbf{2 , 1 5}\end{array}$ & - & $\begin{array}{l}0,93 \\
1,19\end{array}$ & $\begin{array}{l}0,84 \\
\mathbf{4 , 0 0}\end{array}$ & - & $\begin{array}{l}0,27 \\
\mathbf{1 , 0 5}\end{array}$ & - & $\begin{array}{l}0,87 \\
\mathbf{0 , 9 2}\end{array}$ & $\begin{array}{l}2,87 \\
7,81\end{array}$ \\
\hline $\begin{array}{l}\text { Burmannia capitata } \\
\text { (Walter ex J.F. Gmel.) } \\
\text { Mart. }\end{array}$ & $\begin{array}{l}0,50 \\
\mathbf{4 , 6 3}\end{array}$ & $\begin{array}{l}0,12 \\
\mathbf{0 , 9 8}\end{array}$ & - & - & - & - & - & $\begin{array}{l}0,14 \\
\mathbf{1 , 0 8}\end{array}$ & $\begin{array}{l}0,19 \\
\mathbf{3 , 2 3}\end{array}$ & $\begin{array}{l}0,09 \\
\mathbf{1 , 4 1}\end{array}$ & - & - & $\begin{array}{l}0,17 \\
\mathbf{0 , 9 7}\end{array}$ & $\begin{array}{l}0,72 \\
\mathbf{4 , 2 1}\end{array}$ & $\begin{array}{l}0,07 \\
1,56\end{array}$ & - & $\begin{array}{l}0,17 \\
\mathbf{1 , 5 6}\end{array}$ \\
\hline CONVOLVULACEAE & & & & & & & & & & & & & & & & & \\
\hline Cuscuta partita Choisy & $\begin{array}{c}15,72 \\
\mathbf{9 , 2 6}\end{array}$ & $\begin{array}{l}2,81 \\
\mathbf{9 , 8 0}\end{array}$ & $\begin{array}{l}2,52 \\
\mathbf{9 , 0 0}\end{array}$ & $\begin{array}{l}0,44 \\
\mathbf{4 , 0 0}\end{array}$ & $\begin{array}{c}7,97 \\
12,99\end{array}$ & $\begin{array}{c}1,69 \\
\mathbf{1 0 , 2 6}\end{array}$ & $\begin{array}{l}1,42 \\
\mathbf{8 , 3 3}\end{array}$ & $\begin{array}{c}2,31 \\
10,75\end{array}$ & $\begin{array}{l}1,15 \\
\mathbf{8 , 6 0}\end{array}$ & $\begin{array}{l}0,99 \\
\mathbf{7 , 0 4}\end{array}$ & $\begin{array}{l}0,33 \\
\mathbf{4 , 7 6}\end{array}$ & - & $\begin{array}{l}0,92 \\
\mathbf{6 , 8 0}\end{array}$ & $\begin{array}{l}1,00 \\
\mathbf{6 , 3 2}\end{array}$ & $\begin{array}{l}0,59 \\
\mathbf{6 , 2 5}\end{array}$ & $\begin{array}{l}1,46 \\
\mathbf{6 , 4 2}\end{array}$ & $\begin{array}{l}0,23 \\
\mathbf{6 , 2 5}\end{array}$ \\
\hline $\begin{array}{l}\text { Evolvulus gypsophiloides } \\
\text { Moric. }\end{array}$ & $\begin{array}{l}2,24 \\
\mathbf{6 , 4 8}\end{array}$ & $\begin{array}{l}0,31 \\
\mathbf{0 , 9 8}\end{array}$ & $\begin{array}{l}3,06 \\
\mathbf{5 , 0 0}\end{array}$ & $\begin{array}{c}10,98 \\
\mathbf{6 , 6 7}\end{array}$ & - & $\begin{array}{l}1,37 \\
\mathbf{7 , 6 9}\end{array}$ & $\begin{array}{l}7,40 \\
\mathbf{4 , 7 6}\end{array}$ & - & - & - & - & - & $\begin{array}{l}2,77 \\
2,91\end{array}$ & $\begin{array}{l}2,53 \\
\mathbf{6 , 3 2}\end{array}$ & $\begin{array}{l}0,59 \\
\mathbf{3 , 1 3}\end{array}$ & - & - \\
\hline $\begin{array}{l}\text { Jacquemontia montana } \\
\text { (Moric.) Meins }\end{array}$ & - & - & - & - & - & - & & - & - & - & - & - & - & - & $\begin{array}{l}1,33 \\
\mathbf{3 , 1 3}\end{array}$ & - & - \\
\hline CYPERACEAE & & & & & & & & & & & & & & & & & \\
\hline $\begin{array}{l}\text { Bulbostylis conifera } \\
\text { (Kunth) CB. Clarke }\end{array}$ & - & - & - & - & $\begin{array}{l}6,14 \\
\mathbf{9 , 0 9}\end{array}$ & - & - & - & - & - & - & - & - & - & - & - & - \\
\hline $\begin{array}{l}\text { Eleocharis barrosii } \\
\text { Svenson }\end{array}$ & - & - & - & - & - & - & - & - & $\begin{array}{l}2,61 \\
6,45\end{array}$ & - & - & - & - & - & - & - & - \\
\hline $\begin{array}{l}\text { Rhynchospora barbata } \\
\text { (Vahl) Kunth }\end{array}$ & $\begin{array}{l}8,29 \\
\mathbf{7 , 4 1}\end{array}$ & $\begin{array}{l}12,24 \\
\mathbf{8 , 8 2}\end{array}$ & $\begin{array}{l}0,81 \\
\mathbf{4 , 0 0}\end{array}$ & $\begin{array}{c}8,95 \\
10,67\end{array}$ & $\begin{array}{c}9,02 \\
\mathbf{1 0 , 3 9}\end{array}$ & $\begin{array}{c}7,26 \\
\mathbf{1 1 , 5 4}\end{array}$ & - & $\begin{array}{l}2,31 \\
\mathbf{2 , 1 5}\end{array}$ & $\begin{array}{l}1,78 \\
\mathbf{4 , 3 0}\end{array}$ & $\begin{array}{l}40,90 \\
\mathbf{1 4 , 0 8}\end{array}$ & $\begin{array}{l}47,44 \\
\mathbf{1 0 , 7 1}\end{array}$ & - & $\begin{array}{l}3,44 \\
\mathbf{2 , 9 1}\end{array}$ & $\begin{array}{c}10,59 \\
\mathbf{9 , 4 7}\end{array}$ & $\begin{array}{l}4,57 \\
\mathbf{6 , 2 5}\end{array}$ & $\begin{array}{l}2,11 \\
\mathbf{4 , 5 9}\end{array}$ & $\begin{array}{l}0,73 \\
\mathbf{3 , 1 3}\end{array}$ \\
\hline $\begin{array}{l}\text { Rhynchospora filiformis } \\
\text { Vahl }\end{array}$ & - & - & - & - & - & - & - & $\begin{array}{l}0,95 \\
\mathbf{5 , 3 8}\end{array}$ & - & - & - & - & - & - & - & - & - \\
\hline $\begin{array}{l}\text { Rhynchospora hirsuta } \\
\text { (Vahl) Vahl }\end{array}$ & $\begin{array}{l}4,69 \\
7,41\end{array}$ & $\begin{array}{l}1,12 \\
2,94\end{array}$ & $\begin{array}{l}1,98 \\
\mathbf{4 , 0 0}\end{array}$ & - & - & - & $\begin{array}{l}1,57 \\
2,38\end{array}$ & & - & - & - & - & $\begin{array}{l}6,55 \\
\mathbf{5 , 8 3}\end{array}$ & - & - & - & - \\
\hline $\begin{array}{l}\text { Rhynchospora riparia } \\
\text { (Nees) Boeck }\end{array}$ & $\begin{array}{l}0,65 \\
\mathbf{1 , 8 5}\end{array}$ & $\begin{array}{l}0,69 \\
\mathbf{2 , 9 4}\end{array}$ & $\begin{array}{l}12,77 \\
\mathbf{8 , 0 0}\end{array}$ & $\begin{array}{c}7,97 \\
\mathbf{1 2 , 0 0}\end{array}$ & $\begin{array}{l}19,74 \\
\mathbf{1 2 , 9 9}\end{array}$ & $\begin{array}{c}6,21 \\
\mathbf{1 2 , 8 2}\end{array}$ & $\begin{array}{l}7,92 \\
\mathbf{8 , 3 3}\end{array}$ & $\begin{array}{l}2,37 \\
\mathbf{4 , 3 0}\end{array}$ & - & - & - & - & $\begin{array}{c}13,01 \\
\mathbf{8 , 7 4}\end{array}$ & $\begin{array}{l}1,81 \\
\mathbf{3 , 1 6}\end{array}$ & $\begin{array}{l}3,90 \\
4,69\end{array}$ & - & - \\
\hline $\begin{array}{l}\text { Rhynchospora rugosa } \\
\text { (Vahl) Gale }\end{array}$ & - & - & - & - & - & - & - & - & $\begin{array}{l}6,11 \\
5,38\end{array}$ & - & - & - & - & - & - & $\begin{array}{l}2,26 \\
\mathbf{3 , 6 7}\end{array}$ & $\begin{array}{l}0,34 \\
\mathbf{3 , 1 3}\end{array}$ \\
\hline Scleria leptostachya Kunth & - & $\begin{array}{l}0,06 \\
\mathbf{0 , 9 8}\end{array}$ & - & - & - & $\begin{array}{c}4,92 \\
12,82\end{array}$ & $\begin{array}{c}2,84 \\
10,71\end{array}$ & $\begin{array}{l}18,18 \\
\mathbf{1 0 , 7 5}\end{array}$ & - & - & $\begin{array}{l}0,60 \\
\mathbf{3 , 5 7}\end{array}$ & - & - & - & - & - & - \\
\hline $\begin{array}{l}\text { Scleria reticularis Michx. } \\
\text { ex Willd. }\end{array}$ & - & - & - & - & - & - & & $\begin{array}{l}0,27 \\
\mathbf{1 , 0 8}\end{array}$ & - & - & - & $\begin{array}{c}28,52 \\
\mathbf{2 0 , 0}\end{array}$ & $\begin{array}{l}0,25 \\
\mathbf{0 , 9 7}\end{array}$ & - & - & - & - \\
\hline DROSERACEAE & & & & & & & & & & & & & & & & & \\
\hline $\begin{array}{l}\text { Drosera sessilifolia A. } \\
\text { St.-Hil. }\end{array}$ & $\begin{array}{l}1,73 \\
\mathbf{8 , 3 3}\end{array}$ & $\begin{array}{l}0,56 \\
\mathbf{6 , 8 6}\end{array}$ & $\begin{array}{l}0,45 \\
\mathbf{4 , 0 0}\end{array}$ & $\begin{array}{l}0,09 \\
\mathbf{1 , 3 3}\end{array}$ & - & - & - & - & $\begin{array}{l}0,38 \\
\mathbf{2 , 1 5}\end{array}$ & $\begin{array}{c}3,68 \\
\mathbf{1 4 , 0 8}\end{array}$ & $\begin{array}{l}0,77 \\
\mathbf{5 , 9 5}\end{array}$ & - & $\begin{array}{l}0,92 \\
\mathbf{4 , 8 5}\end{array}$ & $\begin{array}{l}0,36 \\
\mathbf{4 , 2 1}\end{array}$ & - & - & - \\
\hline $\begin{array}{l}\text { ERIOCAULACEAE } \\
\text { Paepalanthus manicatus } \\
\text { V.A.Pouls ex Malme. }\end{array}$ & - & - & - & - & - & $\begin{array}{l}0,08 \\
\mathbf{1 , 2 8}\end{array}$ & $\begin{array}{l}0,60 \\
\mathbf{5 , 9 5}\end{array}$ & - & $\begin{array}{l}0,89 \\
\mathbf{5 , 3 8}\end{array}$ & - & - & - & $\begin{array}{l}0,92 \\
\mathbf{3 , 8 8}\end{array}$ & - & - & $\begin{array}{l}1,24 \\
\mathbf{5 , 5 0} \\
\end{array}$ & $\begin{array}{l}0,34 \\
\mathbf{4 , 6 9} \\
\end{array}$ \\
\hline
\end{tabular}




\begin{tabular}{|c|c|c|c|c|c|c|c|c|c|c|c|c|c|c|c|c|c|}
\hline \multirow[t]{2}{*}{ Família/espécie } & \multicolumn{5}{|c|}{$\begin{array}{l}\text { C. limpo } 1 \\
\text { (L1-L5) }\end{array}$} & \multicolumn{3}{|c|}{$\begin{array}{l}\text { C. limpo } 2 \\
\text { (L6-L8) }\end{array}$} & \multicolumn{3}{|c|}{$\begin{array}{l}\text { C. limpo } 3 \\
\text { (L9-L11) }\end{array}$} & \multicolumn{2}{|c|}{$\begin{array}{l}\text { C. limpo } 4 \\
\text { (L12-L13) }\end{array}$} & \multicolumn{2}{|c|}{$\begin{array}{l}\text { C. limpo } 5 \\
\text { (L14-L15) }\end{array}$} & \multicolumn{2}{|c|}{$\begin{array}{l}\text { C. limpo } 6 \\
(\text { L16-L17) }\end{array}$} \\
\hline & L1 & L2 & L3 & L4 & $\mathbf{L 5}$ & L6 & L7 & L8 & L9 & L10 & L11 & L12 & L13 & L14 & L15 & L16 & $\mathbf{L 1 7}$ \\
\hline $\begin{array}{l}\text { Syngonanthus cf. gracilis } \\
\text { (Bong.) Ruhland }\end{array}$ & - & - & - & - & - & - & - & - & - & - & - & - & - & - & - & $\begin{array}{l}0,87 \\
\mathbf{1 , 8 3}\end{array}$ & - \\
\hline $\begin{array}{l}\text { Syngonanthus cf. nitens } \\
\text { (Bong.) Ruhland }\end{array}$ & - & - & - & - & - & - & - & - & - & - & - & - & $\begin{array}{l}1,93 \\
7,77\end{array}$ & - & - & $\begin{array}{l}1,31 \\
\mathbf{3 , 6 7}\end{array}$ & - \\
\hline Syngonanthus sp. & $\begin{array}{l}1,23 \\
\mathbf{6 , 4 8}\end{array}$ & $\begin{array}{l}3,69 \\
\mathbf{5 , 8 8}\end{array}$ & $\begin{array}{l}0,45 \\
\mathbf{4 , 0 0}\end{array}$ & $\begin{array}{l}0,18 \\
\mathbf{2 , 6 7}\end{array}$ & $\begin{array}{l}0,13 \\
\mathbf{1 , 3 0}\end{array}$ & - & - & - & - & - & - & - & - & $\begin{array}{l}1,27 \\
\mathbf{5 , 2 6}\end{array}$ & - & $\begin{array}{l}0,29 \\
\mathbf{0 , 9 2}\end{array}$ & - \\
\hline $\begin{array}{l}\text { EUPHORBIACEAE } \\
\text { Croton pedicellatus Kunth. }\end{array}$ & $\begin{array}{l}0,07 \\
0,93\end{array}$ & $\begin{array}{l}0,19 \\
\mathbf{2 , 9 4}\end{array}$ & - & - & - & $\begin{array}{l}0,08 \\
1,28\end{array}$ & $\begin{array}{l}2,54 \\
1,19\end{array}$ & $\begin{array}{l}2,58 \\
\mathbf{6 , 4 5}\end{array}$ & - & - & $\begin{array}{l}3,90 \\
\mathbf{4 , 7 6}\end{array}$ & $\begin{array}{l}2,31 \\
\mathbf{1 2 , 0}\end{array}$ & - & $\begin{array}{l}0,18 \\
\mathbf{1 , 0 5}\end{array}$ & - & $\begin{array}{l}0,29 \\
\mathbf{0 , 9 2}\end{array}$ & - \\
\hline $\begin{array}{l}\text { FABACEAE } \\
\text { Aeschynomene americana } \\
\text { L. }\end{array}$ & - & - & - & - & - & - & - & - & - & - & $\begin{array}{l}0,22 \\
1,19\end{array}$ & $\begin{array}{l}0,49 \\
\mathbf{2 , 0 0}\end{array}$ & - & - & - & $\begin{array}{l}0,29 \\
\mathbf{0 , 9 2}\end{array}$ & - \\
\hline $\begin{array}{l}\text { Chamaecrista desvauxii } \\
\text { var. chapadicola } \text { H.S.Irwin } \\
\text { \& Barneby }\end{array}$ & - & $\begin{array}{l}0,37 \\
\mathbf{0 , 9 8}\end{array}$ & $\begin{array}{l}0,09 \\
1,00\end{array}$ & $\begin{array}{l}0,18 \\
\mathbf{1 , 3 3}\end{array}$ & - & - & - & - & & $\begin{array}{l}0,09 \\
\mathbf{1 , 4 1}\end{array}$ & $\begin{array}{l}5,06 \\
\mathbf{8 , 3 3}\end{array}$ & $\begin{array}{l}7,15 \\
\mathbf{8 , 0 0}\end{array}$ & - & - & $\begin{array}{l}0,44 \\
\mathbf{3 , 1 3}\end{array}$ & $\begin{array}{l}0,15 \\
\mathbf{0 , 9 2}\end{array}$ & $\begin{array}{l}1,41 \\
\mathbf{4 , 6 9}\end{array}$ \\
\hline $\begin{array}{l}\text { Chamaecrista diphylla (L.) } \\
\text { Greene }\end{array}$ & - & & - & - & - & - & - & - & $\begin{array}{l}5,54 \\
\mathbf{7 , 5 3}\end{array}$ & $\begin{array}{l}0,18 \\
\mathbf{2 , 8 2}\end{array}$ & $\begin{array}{l}0,49 \\
\mathbf{2 , 3 8}\end{array}$ & - & - & - & - & $\begin{array}{l}0,36 \\
\mathbf{0 , 9 2}\end{array}$ & - \\
\hline $\begin{array}{l}\text { Chamaecrista flexuosa (1.) } \\
\text { Greene var. flexuosa }\end{array}$ & - & & - & - & - & - & - & - & - & - & - & $\begin{array}{l}1,26 \\
\mathbf{2 , 0 0}\end{array}$ & $\begin{array}{l}5,21 \\
\mathbf{3 , 8 8}\end{array}$ & $\begin{array}{l}0,18 \\
\mathbf{1 , 0 5}\end{array}$ & $\begin{array}{l}1,62 \\
\mathbf{1 , 5 6}\end{array}$ & $\begin{array}{l}3,86 \\
\mathbf{0 , 9 2}\end{array}$ & $\begin{array}{l}1,74 \\
\mathbf{3 , 1 2}\end{array}$ \\
\hline $\begin{array}{l}\text { Chamaecrista linearis } \\
\text { H.S.Irwin \& Barneby) } \\
\text { Fernandes \& Nunes var. } \\
\text { modesta }\end{array}$ & - & & - & - & - & - & $\begin{array}{l}0,75 \\
\mathbf{2 , 3 8}\end{array}$ & - & - & - & - & - & - & - & - & - & - \\
\hline Mimosa hypoglauca Mart. & $\begin{array}{l}0,43 \\
\mathbf{0 , 9 3}\end{array}$ & $\begin{array}{l}1,31 \\
\mathbf{2 , 9 4}\end{array}$ & $\begin{array}{l}1,17 \\
\mathbf{4 , 0 0}\end{array}$ & $\begin{array}{l}2,13 \\
\mathbf{5 , 3 3}\end{array}$ & $\begin{array}{l}1,31 \\
\mathbf{5 , 1 9}\end{array}$ & - & - & $\begin{array}{l}0,61 \\
\mathbf{1 , 0 8}\end{array}$ & $\begin{array}{c}18,91 \\
\mathbf{8 , 6 0}\end{array}$ & $\begin{array}{l}3,23 \\
\mathbf{8 , 4 5}\end{array}$ & $\begin{array}{l}2,09 \\
\mathbf{4 , 7 6}\end{array}$ & & $\begin{array}{l}1,09 \\
\mathbf{1 , 9 4}\end{array}$ & - & $\begin{array}{l}0,81 \\
\mathbf{4 , 6 9}\end{array}$ & $\begin{array}{l}5,69 \\
7,34\end{array}$ & - \\
\hline $\begin{array}{l}\text { Mimosa somnians Humb. } \\
\text { \& Bonpl. ex Willd }\end{array}$ & - & - & - & - & - & - & - & $\begin{array}{l}1,22 \\
\mathbf{3 , 2 3}\end{array}$ & - & - & $\begin{array}{l}0,38 \\
\mathbf{2 , 3 8}\end{array}$ & $\begin{array}{l}1,54 \\
\mathbf{2 , 0 0}\end{array}$ & - & - & - & - & - \\
\hline $\begin{array}{l}\text { Stylosanthes angustifolia } \\
\text { Vogel }\end{array}$ & $\begin{array}{l}2,09 \\
\mathbf{7 , 4 1}\end{array}$ & $\begin{array}{l}0,12 \\
\mathbf{1 , 9 6}\end{array}$ & $\begin{array}{l}0,81 \\
\mathbf{4 , 0 0}\end{array}$ & $\begin{array}{l}0,53 \\
\mathbf{4 , 0 0}\end{array}$ & $\begin{array}{c}4,97 \\
\mathbf{1 0 , 3 9}\end{array}$ & $\begin{array}{l}0,32 \\
\mathbf{5 , 1 3}\end{array}$ & $\begin{array}{l}0,07 \\
\mathbf{1 , 1 9}\end{array}$ & $\begin{array}{l}0,20 \\
\mathbf{2 , 1 5}\end{array}$ & $\begin{array}{l}0,32 \\
\mathbf{2 , 1 5}\end{array}$ & $\begin{array}{l}18,92 \\
\mathbf{1 4 , 0 8}\end{array}$ & $\begin{array}{l}3,63 \\
\mathbf{9 , 5 2}\end{array}$ & - & $\begin{array}{l}2,52 \\
\mathbf{5 , 8 3}\end{array}$ & $\begin{array}{l}3,71 \\
\mathbf{8 , 4 2}\end{array}$ & $\begin{array}{l}0,96 \\
\mathbf{4 , 6 9}\end{array}$ & $\begin{array}{l}1,24 \\
\mathbf{6 , 4 2}\end{array}$ & - \\
\hline $\begin{array}{l}\text { GENTIANACEAE } \\
\text { Curtia tenella (Mart.) } \\
\text { Cham }\end{array}$ & $\begin{array}{l}0,07 \\
\mathbf{0 , 9 3}\end{array}$ & - & - & - & - & - & - & - & - & - & - & - & - & $\begin{array}{l}0,63 \\
\mathbf{6 , 3 2}\end{array}$ & $\begin{array}{l}0,15 \\
\mathbf{1 , 5 6}\end{array}$ & - & - \\
\hline $\begin{array}{l}\text { Curtia tenuifolia (Aubl.) } \\
\text { Knobl. }\end{array}$ & $\begin{array}{l}0,29 \\
\mathbf{2 , 7 8}\end{array}$ & - & $\begin{array}{l}0,81 \\
\mathbf{4 , 0 0}\end{array}$ & - & - & - & - & - & - & - & - & - & - & - & - & - & - \\
\hline $\begin{array}{l}\text { KRAMERIACEAE } \\
\text { Krameria grandiflora } \\
\text { A.St.-Hil. } \\
\text { LAMIACEAE }\end{array}$ & - & - & - & - & $\begin{array}{l}0,13 \\
\mathbf{1 , 3 0}\end{array}$ & - & & - & - & - & - & $\begin{array}{l}0,91 \\
\mathbf{8 , 0 0}\end{array}$ & - & - & $\begin{array}{l}0,07 \\
1,56\end{array}$ & - & - \\
\hline Eriope sp. & $\begin{array}{l}1,37 \\
\mathbf{3 , 7 0}\end{array}$ & - & $\begin{array}{l}0,09 \\
\mathbf{1 , 0 0}\end{array}$ & - & $\begin{array}{l}5,23 \\
\mathbf{6 , 4 9}\end{array}$ & - & - & - & - & - & - & $\begin{array}{l}0,21 \\
\mathbf{2 , 0 0}\end{array}$ & $\begin{array}{l}4,87 \\
\mathbf{3 , 8 8}\end{array}$ & $\begin{array}{l}1,99 \\
\mathbf{2 , 1 1}\end{array}$ & $\begin{array}{c}5,82 \\
14,06\end{array}$ & - & - \\
\hline Hyptis atrorubens Poit. & - & - & - & - & - & - & $\begin{array}{l}0,52 \\
\mathbf{3 , 5 7}\end{array}$ & $\begin{array}{l}0,20 \\
\mathbf{2 , 1 5}\end{array}$ & - & - & $\begin{array}{l}2,09 \\
\mathbf{3 , 5 7}\end{array}$ & $\begin{array}{l}0,21 \\
\mathbf{2 , 0 0}\end{array}$ & - & - & - & $\begin{array}{l}0,22 \\
\mathbf{0 , 9 2}\end{array}$ & $\begin{array}{l}1,18 \\
\mathbf{4 , 6 9}\end{array}$ \\
\hline $\begin{array}{l}\text { Hyptis crenata } \text { Pohl \& } \\
\text { Benth. } \\
\text { LENTIBULARIACEAE }\end{array}$ & - & - & - & - & - & $\begin{array}{l}0,08 \\
\mathbf{1 , 2 8}\end{array}$ & $\begin{array}{l}0,90 \\
\mathbf{3 , 5 7}\end{array}$ & $\begin{array}{l}2,71 \\
\mathbf{2 , 1 5}\end{array}$ & - & - & - & - & - & - & - & - & - \\
\hline Utricularia simulans Pilg. & - & - & - & - & - & - & - & - & $\begin{array}{l}0,19 \\
\mathbf{1 , 0 8}\end{array}$ & $\begin{array}{l}0,09 \\
\mathbf{1 , 4 1}\end{array}$ & - & & - & - & - & - & - \\
\hline Utricularia subulata L. & - & - & - & - & - & - & - & - & $\begin{array}{l}3,31 \\
\mathbf{8 , 6 0}\end{array}$ & $\begin{array}{l}0,18 \\
\mathbf{2 , 8 2}\end{array}$ & - & $\begin{array}{l}0,07 \\
\mathbf{2 , 0 0}\end{array}$ & - & - & - & - & - \\
\hline LYTHRACEAE & & & & & & & & & & & & & & & & & \\
\hline Cuphea laricoides Koehne & - & - & - & - & $\begin{array}{l}3,92 \\
1,30\end{array}$ & - & - & - & - & - & $\begin{array}{l}0,16 \\
1,19\end{array}$ & - & - & - & - & - & - \\
\hline MALVACEAE & & & & & & & & & & & & & & & & & \\
\hline Sida linifolia Cav. & - & - & - & - & - & - & $\begin{array}{l}0,22 \\
\mathbf{3 , 5 7}\end{array}$ & - & - & - & - & - & - & - & - & - & - \\
\hline Sida viarum A. St.-Hil. & - & $\begin{array}{l}0,12 \\
\mathbf{0 , 9 8}\end{array}$ & - & - & - & - & - & - & - & - & - & - & - & - & - & - & - \\
\hline MELASTOMATACEAE & & & & & & & & & & & & & & & & & \\
\hline $\begin{array}{l}\text { Acisanthera bivalvis } \\
\text { (Aubl.) Cogn. }\end{array}$ & - & - & - & - & - & $\begin{array}{l}0,73 \\
7,69\end{array}$ & $\begin{array}{l}0,75 \\
\mathbf{8 , 3 3}\end{array}$ & $\begin{array}{l}2,71 \\
\mathbf{7 , 5 3}\end{array}$ & - & - & - & - & $\begin{array}{l}0,08 \\
\mathbf{0 , 9 7}\end{array}$ & - & - & - & - \\
\hline Acisanthera fluitans Cogn. & - & - & - & - & - & - & - & - & - & - & - & - & $\begin{array}{l}2,10 \\
\mathbf{3 , 8 8}\end{array}$ & - & - & - & $\begin{array}{l}0,51 \\
4,69\end{array}$ \\
\hline $\begin{array}{l}\text { Rhynchanthera grandiflora } \\
\text { (Aubl.) DC. } \\
\text { OCHNACEAE }\end{array}$ & - & - & - & - & - & - & - & - & $\begin{array}{l}34,12 \\
\mathbf{1 0 , 7 5}\end{array}$ & - & $\begin{array}{l}4,95 \\
\mathbf{5 , 9 5}\end{array}$ & - & - & - & - & $\begin{array}{c}11,66 \\
7,34\end{array}$ & $\begin{array}{l}50,37 \\
\mathbf{1 5 , 6 3}\end{array}$ \\
\hline Sauvagesia erecta L. & - & - & - & - & - & $\begin{array}{l}1,77 \\
\mathbf{6 , 4 1}\end{array}$ & $\begin{array}{l}0,60 \\
\mathbf{3 , 5 7}\end{array}$ & $\begin{array}{l}0,41 \\
\mathbf{4 , 3 0}\end{array}$ & - & - & - & $\begin{array}{l}0,56 \\
\mathbf{8 , 0 0}\end{array}$ & - & - & - & - & - \\
\hline
\end{tabular}




\begin{tabular}{|c|c|c|c|c|c|c|c|c|c|c|c|c|c|c|c|c|c|}
\hline \multirow[t]{2}{*}{ Família/espécie } & \multicolumn{5}{|c|}{$\begin{array}{l}\text { C. limpo } 1 \\
\text { (L1-L5) }\end{array}$} & \multicolumn{3}{|c|}{$\begin{array}{l}\text { C. limpo } 2 \\
\text { (L6-L8) }\end{array}$} & \multicolumn{3}{|c|}{$\begin{array}{l}\text { C. limpo } 3 \\
\text { (L9-L11) }\end{array}$} & \multicolumn{2}{|c|}{$\begin{array}{l}\text { C. limpo } 4 \\
\text { (L12-L13) }\end{array}$} & \multicolumn{2}{|c|}{$\begin{array}{l}\text { C. limpo } 5 \\
\text { (L14-L15) }\end{array}$} & \multicolumn{2}{|c|}{$\begin{array}{l}\text { C. limpo } 6 \\
\text { (L16-L17) }\end{array}$} \\
\hline & L1 & L2 & L3 & L4 & L5 & L6 & L7 & L8 & L9 & L10 & L11 & L12 & L13 & L14 & L15 & L16 & L17 \\
\hline $\begin{array}{l}\text { ORCHIDACEAE } \\
\text { Habenaria spathulifera } \\
\text { Cogn. } \\
\text { OXALIDACEAE }\end{array}$ & - & - & - & - & - & - & & $\begin{array}{l}0,14 \\
\mathbf{1 , 0 8}\end{array}$ & - & - & $\begin{array}{l}0,38 \\
\mathbf{1 , 1 9}\end{array}$ & - & - & - & - & - & - \\
\hline $\begin{array}{l}\text { Oxalis divaricata Mart. } \\
\text { ex Zucc. } \\
\text { PASSIFLORACEAE }\end{array}$ & - & - & - & - & - & - & $\begin{array}{l}0,15 \\
\mathbf{2 , 3 8}\end{array}$ & $\begin{array}{l}0,07 \\
\mathbf{1 , 0 8}\end{array}$ & - & - & $\begin{array}{l}0,05 \\
1,19\end{array}$ & - & & - & $\begin{array}{l}0,22 \\
\mathbf{1 , 5 6}\end{array}$ & & - \\
\hline Piriqueta plicata Urb. & - & - & - & - & - & $\begin{array}{l}0,08 \\
1,28\end{array}$ & $\begin{array}{l}0,90 \\
\mathbf{4 , 7 6}\end{array}$ & $\begin{array}{l}0,27 \\
\mathbf{1 , 0 8}\end{array}$ & - & - & - & - & - & - & - & - & - \\
\hline Turnera caerulea DC. & - & - & - & - & - & - & - & - & - & - & - & - & - & - & - & $\begin{array}{l}1,24 \\
\mathbf{5 , 5 0}\end{array}$ & - \\
\hline $\begin{array}{l}\text { Turnera oblongifolia } \\
\text { Cambess. } \\
\text { PHYLLANTHACEAE }\end{array}$ & - & $\begin{array}{l}2,00 \\
\mathbf{4 , 9 0}\end{array}$ & $\begin{array}{l}0,99 \\
\mathbf{4 , 0 0}\end{array}$ & $\begin{array}{l}0,44 \\
\mathbf{2 , 6 7}\end{array}$ & - & - & - & - & - & - & - & - & - & - & - & - & - \\
\hline Phyllanthus sp. & - & - & - & - & - & - & - & - & - & - & - & - & - & - & - & $\begin{array}{l}0,15 \\
\mathbf{0 , 9 2}\end{array}$ & - \\
\hline $\begin{array}{l}\text { PLANTAGINACEAE } \\
\text { Bacopa angulata (Benth.) } \\
\text { Edwall }\end{array}$ & - & - & - & & - & - & - & - & $\begin{array}{l}0,06 \\
1,08\end{array}$ & - & - & - & - & - & - & - & - \\
\hline $\begin{array}{l}\text { POACEAE } \\
\text { Axonopus purpusii (Mez) } \\
\text { Chase }\end{array}$ & - & - & $\begin{array}{c}30,22 \\
\mathbf{6 , 0 0}\end{array}$ & $\begin{array}{c}12,49 \\
\mathbf{4 , 0 0}\end{array}$ & - & - & - & - & - & - & - & - & - & - & - & - & - \\
\hline $\begin{array}{l}\text { Mesosetum loliiforme } \\
\text { (Hochst. ex Steud.) Chase }\end{array}$ & - & - & - & - & $\begin{array}{l}24,97 \\
10,39\end{array}$ & - & - & - & $\begin{array}{l}6,17 \\
\mathbf{7 , 5 3}\end{array}$ & $\begin{array}{l}12,20 \\
\mathbf{8 , 4 5}\end{array}$ & $\begin{array}{l}10,39 \\
\mathbf{3 , 5 7}\end{array}$ & - & - & - & - & $\begin{array}{c}33,09 \\
\mathbf{9 , 1 7}\end{array}$ & $\begin{array}{l}24,85 \\
\mathbf{1 5 , 6 3}\end{array}$ \\
\hline Mesosetum sp. nov. & - & - & $\begin{array}{c}20,41 \\
\mathbf{5 , 0 0}\end{array}$ & $\begin{array}{c}26,75 \\
\mathbf{9 , 3 3}\end{array}$ & - & - & - & - & - & - & - & - & - & - & - & - & - \\
\hline Paspalum multicaule Poir & $\begin{array}{l}2,74 \\
\mathbf{2 , 7 8}\end{array}$ & $\begin{array}{l}17,36 \\
\mathbf{8 , 8 2}\end{array}$ & $\begin{array}{l}3,96 \\
\mathbf{3 , 0 0}\end{array}$ & $\begin{array}{l}0,71 \\
1,33\end{array}$ & $\begin{array}{l}0,26 \\
1,30\end{array}$ & - & - & - & - & $\begin{array}{l}0,36 \\
\mathbf{1 , 4 1}\end{array}$ & - & - & $\begin{array}{l}2,10 \\
\mathbf{4 , 8 5}\end{array}$ & $\begin{array}{l}4,98 \\
\mathbf{4 , 2 1}\end{array}$ & $\begin{array}{l}4,79 \\
\mathbf{6 , 2 5}\end{array}$ & & - \\
\hline $\begin{array}{l}\text { Sacciolepis vilvoides } \\
\text { (Trin.) Chase }\end{array}$ & - & - & - & - & - & - & - & - & - & - & - & - & - & - & - & $\begin{array}{c}16,11 \\
\mathbf{6 , 4 2}\end{array}$ & $\begin{array}{l}14,14 \\
\mathbf{1 5 , 6 3}\end{array}$ \\
\hline $\begin{array}{l}\text { Steirachne barbata (Trin.) } \\
\text { Renvoize }\end{array}$ & $\begin{array}{l}0,65 \\
\mathbf{2 , 7 8}\end{array}$ & $\begin{array}{l}0,37 \\
\mathbf{3 , 9 2}\end{array}$ & $\begin{array}{l}0,72 \\
\mathbf{3 , 0 0}\end{array}$ & $\begin{array}{l}1,06 \\
\mathbf{6 , 6 7}\end{array}$ & $\begin{array}{l}15,42 \\
\mathbf{1 2 , 9 9}\end{array}$ & - & - & - & $\begin{array}{c}15,72 \\
\mathbf{9 , 6 8}\end{array}$ & $\begin{array}{l}18,30 \\
\mathbf{1 4 , 0 8}\end{array}$ & $\begin{array}{c}10,17 \\
\mathbf{7 , 1 4}\end{array}$ & - & $\begin{array}{l}0,08 \\
\mathbf{0 , 9 7}\end{array}$ & $\begin{array}{l}6,97 \\
7,37\end{array}$ & $\begin{array}{l}2,80 \\
\mathbf{7 , 8 1}\end{array}$ & $\begin{array}{l}5,25 \\
\mathbf{6 , 4 2}\end{array}$ & - \\
\hline $\begin{array}{l}\text { Trachypogon spicatus (L. } \\
\text { f.) Kuntze }\end{array}$ & $\begin{array}{c}53,57 \\
\mathbf{9 , 2 6}\end{array}$ & $\begin{array}{c}48,34 \\
\mathbf{9 , 8 0}\end{array}$ & $\begin{array}{c}14,84 \\
\mathbf{5 , 0 0}\end{array}$ & $\begin{array}{l}23,29 \\
10,67\end{array}$ & & $\begin{array}{l}73,95 \\
12,82\end{array}$ & $\begin{array}{l}63,23 \\
\mathbf{1 0 , 7 1}\end{array}$ & $\begin{array}{l}49,12 \\
\mathbf{1 0 , 7 5}\end{array}$ & - & - & - & $\begin{array}{c}54,87 \\
\mathbf{2 0 , 0}\end{array}$ & $\begin{array}{c}43,58 \\
\mathbf{9 , 7 1}\end{array}$ & $\begin{array}{l}50,50 \\
\mathbf{9 , 4 7}\end{array}$ & $\begin{array}{l}69,00 \\
\mathbf{1 5 , 6 3}\end{array}$ & & - \\
\hline $\begin{array}{l}\text { POLYGALACEAE } \\
\text { Polygala celosioides Mart. }\end{array}$ & $\begin{array}{l}0,07 \\
\mathbf{0 , 9 3}\end{array}$ & - & - & - & - & - & - & - & - & - & $\begin{array}{l}0,05 \\
1,19\end{array}$ & - & - & $\begin{array}{l}1,09 \\
\mathbf{2 , 1 1}\end{array}$ & $\begin{array}{l}0,07 \\
1,56\end{array}$ & - & - \\
\hline $\begin{array}{l}\text { Polygala sedoides A. W. } \\
\text { Benn }\end{array}$ & $\begin{array}{l}0,14 \\
\mathbf{0 , 9 3}\end{array}$ & $\begin{array}{l}0,25 \\
\mathbf{0 , 9 8}\end{array}$ & $\begin{array}{l}0,27 \\
\mathbf{3 , 0 0}\end{array}$ & $\begin{array}{l}0,18 \\
\mathbf{2 , 6 7}\end{array}$ & - & - & - & - & - & $\begin{array}{l}0,27 \\
\mathbf{2 , 8 2}\end{array}$ & $\begin{array}{l}0,05 \\
1,19\end{array}$ & - & - & - & - & - & - \\
\hline Polygala subtilis Kunth & - & - & - & - & - & - & - & - & $\begin{array}{l}0,25 \\
\mathbf{2 , 1 5}\end{array}$ & - & $\begin{array}{l}0,16 \\
\mathbf{2 , 3 8}\end{array}$ & - & - & - & - & - & - \\
\hline Polygala longicaulis Kunth & - & - & - & - & - & $\begin{array}{l}0,08 \\
\mathbf{1 , 2 8}\end{array}$ & - & - & - & - & - & - & - & - & - & - & - \\
\hline $\begin{array}{l}\text { RUBIACEAE } \\
\text { Borreria densiflora DC. }\end{array}$ & - & - & - & $\begin{array}{l}0,09 \\
1,33\end{array}$ & - & - & - & - & - & - & - & - & - & - & - & - & - \\
\hline $\begin{array}{l}\text { Borreria scabiosoides } \\
\text { Cham. \& Schltdl. }\end{array}$ & - & - & - & - & - & - & - & - & - & - & - & - & - & - & - & $\begin{array}{l}0,07 \\
\mathbf{0 , 9 2}\end{array}$ & $\begin{array}{l}0,45 \\
\mathbf{3 , 1 3}\end{array}$ \\
\hline $\begin{array}{l}\text { Diodella apiculata (Willd. } \\
\text { ex Roen.\& Schult.) } \\
\text { Delprete }\end{array}$ & $\begin{array}{l}0,58 \\
\mathbf{2 , 7 8}\end{array}$ & $\begin{array}{l}0,25 \\
\mathbf{1 , 9 6}\end{array}$ & $\begin{array}{l}0,18 \\
\mathbf{1 , 0 0}\end{array}$ & - & $\begin{array}{l}0,13 \\
1,30\end{array}$ & - & - & - & - & - & $\begin{array}{l}0,16 \\
\mathbf{2 , 3 8}\end{array}$ & - & - & - & - & - & - \\
\hline $\begin{array}{l}\text { Limnosipanea } \\
\text { schomburgkii Hook. f. } \\
\text { XYRIDACEAE }\end{array}$ & - & - & - & - & & - & $\begin{array}{l}0,15 \\
\mathbf{1 , 1 9}\end{array}$ & - & $\begin{array}{l}0,25 \\
\mathbf{3 , 2 3}\end{array}$ & - & $\begin{array}{l}0,16 \\
\mathbf{2 , 3 8}\end{array}$ & - & - & - & - & $\begin{array}{l}1,17 \\
\mathbf{6 , 4 2}\end{array}$ & $\begin{array}{l}0,11 \\
\mathbf{3 , 1 3}\end{array}$ \\
\hline Xyris guianensis Steudel & - & - & - & - & - & - & $\begin{array}{l}0,07 \\
\mathbf{1 , 1 9}\end{array}$ & $\begin{array}{l}6,99 \\
\mathbf{7 , 5 3}\end{array}$ & - & - & - & & - & - & - & - & - \\
\hline Xyris jupicai Rich & - & - & - & - & - & - & - & - & - & - & - & $\begin{array}{l}0,14 \\
\mathbf{2 , 0 0}\end{array}$ & - & - & - & $\begin{array}{l}0,44 \\
\mathbf{3 , 6 7}\end{array}$ & $\begin{array}{l}0,56 \\
\mathbf{3 , 1 3}\end{array}$ \\
\hline $\begin{array}{l}\text { Xyris paraensis Poeppig } \\
\text { ex Kunth }\end{array}$ & $\begin{array}{l}0,07 \\
\mathbf{0 , 9 3}\end{array}$ & - & $\begin{array}{l}0,36 \\
\mathbf{2 , 0 0}\end{array}$ & - & - & - & - & - & - & - & - & - & $\begin{array}{l}3,02 \\
7,77\end{array}$ & - & - & $\begin{array}{l}8,31 \\
\mathbf{6 , 4 2}\end{array}$ & - \\
\hline Xyris savanensis Miq. & $\begin{array}{l}0,36 \\
\mathbf{3 , 7 0}\end{array}$ & $\begin{array}{l}2,25 \\
\mathbf{6 , 8 6}\end{array}$ & $\begin{array}{l}2,07 \\
\mathbf{8 , 0 0}\end{array}$ & $\begin{array}{c}3,54 \\
13,33\end{array}$ & - & - & $\begin{array}{l}0,07 \\
\mathbf{1 , 1 9}\end{array}$ & $\begin{array}{l}0,34 \\
\mathbf{3 , 2 3}\end{array}$ & - & $\begin{array}{l}0,54 \\
\mathbf{5 , 6 3}\end{array}$ & $\begin{array}{l}0,33 \\
\mathbf{4 , 7 6}\end{array}$ & - & $\begin{array}{l}0,08 \\
\mathbf{0 , 9 7}\end{array}$ & $\begin{array}{l}1,63 \\
\mathbf{7 , 3 7}\end{array}$ & - & - & - \\
\hline $\begin{array}{l}\text { Número de espécies por } \\
\text { linha }\end{array}$ & 24 & 23 & 24 & 18 & 16 & 15 & 21 & 22 & 19 & 15 & 26 & 15 & 22 & 19 & 19 & 24 & 16 \\
\hline $\mathrm{N}^{0}$ de espécies por área & 35 & & & & & 29 & & & 34 & & & 32 & & 24 & & 28 & \\
\hline $\begin{array}{l}\text { H' }^{\prime}(\text { nats.cobertura } \\
\text { área }\end{array}$ & 2,5 & & & & & 1,63 & & & 2,32 & & & 1,98 & & 1,65 & & 1,97 & \\
\hline $\begin{array}{l}\mathrm{H}^{\prime}(\text { nats.cobertura } \\
\\
\text { as linhas }\end{array}$ & 2,84 & & & & & & & & & & & & & & & & \\
\hline
\end{tabular}

